\title{
CO observations and investigation of triggered star formation towards N10 infrared bubble and surroundings
}

\author{
D.R.G. Gama, J.R.D. Lepine and E. Mendoza \\ Departamento de Astronomia do IAG/USP, Sao Paulo - Brazil \\ Y. Wu \\ University of Peking, Beijing - China \\ ywu@pku.edu.cn \\ and \\ J. Yuan \\ National Astronomical Observatories of China (NAOC)
}

\begin{abstract}
We studied the environment of the dust bubble N10 in molecular emission. Infrared bubbles, first detected by the GLIMPSE survey at $8.0 \mu \mathrm{m}$, are ideal regions to investigate the effect of the expansion of the HII region on its surroundings eventual triggered star formation at its borders. In this work, we present a multi-wavelength study of N10. This bubble is especially interesting as infrared studies of the young stellar content suggest a scenario of ongoing star formation, possibly triggered, on the edge of the HII region. We carried out observations of ${ }^{12} \mathrm{CO}(1-0)$ and ${ }^{13} \mathrm{CO}(1-0)$ emission at PMO 13.7-m towards N10. We also analyzed the IR and sub-mm emission on this region and compare those different tracers to obtain a detailed view of the interaction between the expanding HII region and the molecular gas. We also estimated the parameters of the denser cold dust condensation and of the ionized gas inside the shell. Bright $\mathrm{CO}$ emission was detected and two molecular clumps were identified, from which we have derived physical parameters. We also estimate the parameters for the densest cold dust condensation and for the ionized gas inside the shell. The comparison between the dynamical age of this region and the fragmentation time scale favors the "Radiation-Driven Implosion" mechanism of star formation. N10 reveals to be specially interesting case with gas structures in a narrow frontier between HII region and surrounding molecular material, and with a range of ages of YSOs situated in region indicating triggered star formation.
\end{abstract}

Subject headings: ISM: bubbles — ISM: HII regions — ISM: molecules — stars: formation

\section{Introduction}

In the last decade the studies about massive star forming regions have gained considerable attention. Questions as whether the interaction of massive stars with their surrounding molecular clouds triggers the star formation have been amply discussed. The discovery of the "infrared bubbles", a new type of object first cataloged through the GLIMPSE1 (Benjamin et al. 2003; Churchwell et al. 2009) at $8.0 \mu \mathrm{m}$, offers a new powerful tool to investigate the star formation process. Those objects present a bright border at $8.0 \mu \mathrm{m}$, caused by the emission of Polycyclic Aromatic Hydrocarbons (PAHs), excited by ultraviolet radiation (UV), which surrounds a region of ionized gas (Churchwell et al. 2006, 2007).

${ }^{1}$ Galactic Legacy Infrared Mid-Plane Survey Extraordinaire 
The bubbles were detected in observations performed by the Spitzer satellite, in a survey that revealed about 600 bright objects at mid-infrared wavelengths (Churchwell et al. 2006). Shortly afterwards the Churchwell catalog were complemented by the Milky Way Project (MWP) catalog by Simpson et al. (2012). This is the most recent scientific citizengenerated catalog, where the bubbles were identified by thousands of volunteers and therefore their classification is more reliable. Deharveng et al. (2010) have identified and studied a large sample of bubbles and concluded that the shell of each one, detected at $8.0 \mu \mathrm{m}$, is an evidence of an HII region produced by the ionizing massive stars. Thompson et al. (2012) suggested that the expansion of the bubbles triggers the formation of Young Stellar Objects (YSOs) which is a non-negligible process in Galactic scales. Kendrew et al. 2012, 2016) also have observed this behavior and, although evidences of triggering via bubble expansion were missing, these authors found populations of YSOs near the borders of expanding bubbles, which could offer us important clues to the star formation process and the expansion of infrared bubbles.

We can interpret these bubbles as basically ionized gas surrounded by cold dust. A Photon-Dominated Region (PDR) in the inner regions of the shell, can be identified and described at mid-infrared wavelengths (Lefloch et al. 2005). The PDRs can be the result of the HII region expansion. They can be seen as regions where the ionization front is still progressing in the densest medium of the original cloud, generating an interface between ionized and neutral gases. The larger density in the PDR is possibly due to material collected by the expansion of the HII region. In this region the UV flux decreases sharply, allowing the existence of molecular and grain species. The massive stars interact with the original molecular cloud and, by their UV radiation, generate the interface between ionized gas and neutral gas.

In principle, the structure of the objects, should it be spherical shells or rings, allow us to understand correctly the kinematics of the gas and the chronology of the newly formed stars. Several works claimed that there was evidence for star formation triggered by the expansion of the HII region (Dewangan et al.2012; Zavagno et al. 2010; Beuther et al. 2011). Yuan et al. (2014) found velocity differences of the order of 30 $\mathrm{km} \mathrm{s}^{-1}$ between distinct parts of the ring in the bubble N6, which would indicate a quite larger expansion ve- locity than those considered by other authors, of a few $\mathrm{km} \mathrm{s}^{-1}$ (e.g. Beaumont \& Williams 2010). Dale et al. (2005) and Dale \& Bonnell (2008) carried out simulations to study the effects of stellar feedback in molecular clouds. They suggested that it is not possible to determine if the formation of an YSO was triggered or not by the expansion of the HII region and as a consequence studies of triggered star formation should be done statistically.

Therefore, it is important to gather a number of well studied bubbles to establish if they have similar formation histories, if they have similar morphology and if they give similar answers to the process about triggered star formation.

In this work we present a detailed study of N10, a remarkable bubble which has molecular clumps and YSOs associated with its surrounding shell. We present our CO observations and we analyze them together with the relevant data at different wavelengths: $8.0 \mu \mathrm{m}$, PAH emission; $24 \mu \mathrm{m}$, hot grains from ionized region; $870 \mu \mathrm{m}$, dust emission; and $20 \mathrm{~cm}$ free-free emission, from hot gas.

This paper is organized as follows: our target object is introduced in Section 2. In Section 3 we describe the $\mathrm{CO}$ observations and the archival data used in this paper. We dedicate the Section 4 to present our results and the Section 5 to discuss these results. Finally, we summarized our mainly conclusions in Section 6

\section{The bubble N10}

$\mathrm{N} 10$ is situated in the direction $l=13.188^{\circ}$, $b=0.039^{\circ}$ (Churchwell et al. 2006). This object is also identified as MWP1G013189+000428 by Simpson et al. (2012). It appears in the SpitzerGLIMPSE $8.0 \mu \mathrm{m}$ image as a bright ring-like structure (Figure 1). The $8.0 \mu \mathrm{m}$ emission in the border of bubbles is attributed to PDRs containing PAHs; the gas density in the PDR can be much larger (up to a factor 10) than that of the surrounding medium (Deharveng et al. 2010; Churchwell et al. 2006). In Figure 1 we show an ellipse to mark the boundaries of the bubble, for later reference in images at different wavelengths. Churchwell et al. (2006) consider N10 a bipolar (or double) bubble, since a small bubble (N11) seems to be connected to N10 in the North of it. However, Deharveng et al. (2015) recently showed that N10/N11 is not a bipolar bubble, as Churchwell et al. (2006) misidentified. In this work we deal only with the case of N10. 


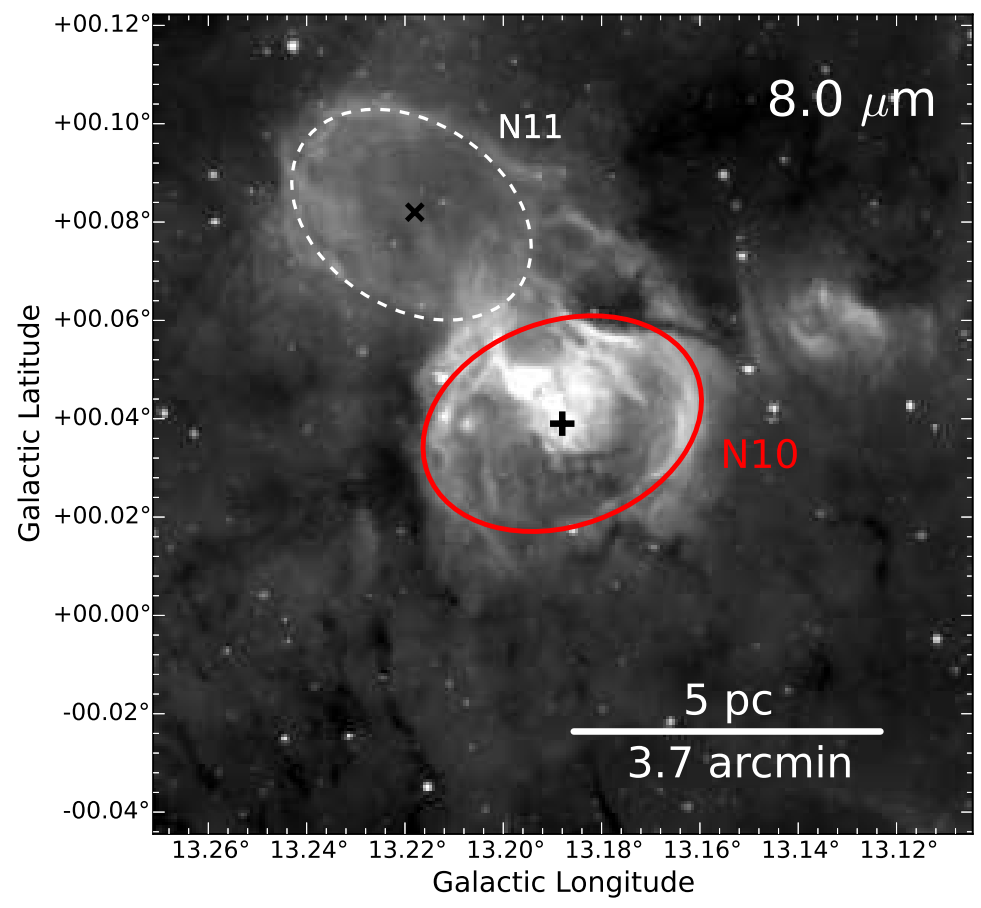

Fig. 1.- Map of the bubble N10, located to a distance of $4.7 \mathrm{kpc}$, at $8.0 \mu \mathrm{m}$. The black cross indicates the center of the HII region inside the bubble of our interest. The red ellipse indicates the edge of N10. The scale corresponds to a field of $5 \mathrm{pc}\left(\sim 3.7^{\prime}\right)$. The white dashed ellipse indicates N11 position, which center is located at $l=13.218^{\circ}, b=0.082^{\circ}$, marked by the black "x".

The distance of N10 was estimated as $4.9 \mathrm{kpc}$ by Churchwell et al. (2006), $4.1 \mathrm{kpc}$ by Beaumont \& Williams (2010), $4.6 \mathrm{kpc}$ by Pandian et al. (2008) and $4.9 \mathrm{kpc}$ by Watson et al. (2008). These are kinematic distances estimated using different rotation curves, which explain the discrepancies.

A methanol $\left(\mathrm{CH}_{3} \mathrm{OH}\right)$ maser in $\mathrm{N} 10$ region was first reported by Szymczak et al. (2000), detected towards the IRAS 18111-1729 source. It is accepted that the methanol masers (as is the case of the present one) are associated with the earliest stages of massive star formation (Minier \& Booth 2002). Figure 2 shows this methanol maser source located on the border of one of the two bright $870 \mu \mathrm{m}$ condensations adjacent to the bubble. The second $\mathrm{CH}_{3} \mathrm{OH}$ maser reported by Pandian et al. (2008) seems to be associated with a SVSS2 2 source.

In their study of the central region, Watson et al. (2008) identified four stars as possible ionizing stars

${ }^{2}$ NRAO VLA Sky Survey (Condon et al. 1998) in N10 (see Table 1), based on their Spectral Energy Distributions (SEDs) which are well-fitted by a stellar photosphere. The position of stars are also plotted in Figure 2. Assuming a radius of $1.61 \mathrm{pc}$ for the densest dark cloud, Ma et al. (2013) estimated a dynamical age $\mathrm{t}_{d y n}=9.17 \times 10^{4} \mathrm{yr}$ for N10. Nevertheless, they argue that this value could be larger since the density of the true ambient where the stars originally were formed could be larger that they considered.

Hereafter we will adopt the position of N10 and the ellipse in Figure 2 as reference: top of the bubble (higher galactic latitude with the center as reference), bottom (lower galactic latitude), right (lower galactic longitude) and left (higher galactic longitude).

\section{Observations and data}

\subsection{CO observations}

The observations were carried out with PMO (Purple Mountain Observatory) $13.7-\mathrm{m}$ radio telescope in 2012 June. We observed the $J=1-0$ tran- 
TABLE 1

CAndidates IONIZING STARs IN N10 System.

\begin{tabular}{ccccc}
\hline \hline ID $^{\text {a }}$ & A.R. (J2000) & DEC (J2000) & Spectral Type & $\mathrm{A}_{V}$ \\
\hline IN10-1 & 181406.343 & -172833.86 & O7.5 V & 7 \\
IN10-2 & 181404.771 & -172758.74 & O6.5 V & 7 \\
IN10-3 & 181407.104 & -172921.27 & O6 V & 5 \\
IN10-4 & 181406.666 & -172921.34 & O7 V & 8 \\
\hline
\end{tabular}

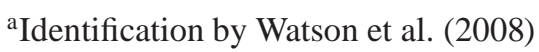

sition of ${ }^{12} \mathrm{CO}(115.27 \mathrm{GHz}),{ }^{13} \mathrm{CO}(110.20 \mathrm{GHz})$ and $\mathrm{C}^{18} \mathrm{O}(109.78 \mathrm{GHz})$. The On-The-Fly (OTF) observing mode was applied to map a $21^{\prime} \times 25^{\prime}$ region centered at $\alpha_{2000}=18^{\mathrm{h}} 14^{\mathrm{m}} 01^{\mathrm{s}} .361$ and $\delta_{2000}=$ $-17 \mathrm{deg} 28^{\prime} 23^{\prime \prime} .14$. For the 13.7-m PMO antenna, we have considered a Half Power Beam Width (HPBW) around $52^{\prime \prime}$.

We used a 9 beam array of SIS receivers at the front end (Shan et al. 2012). The main beam efficiency at the center of the $3 \times 3$ array is about 0.44 at $115 \mathrm{GHz}$ and 0.48 at $110 \mathrm{GHz}$. Our spectral resolution was about $61 \mathrm{kHz}$, corresponding to velocity resolutions of $0.16 \mathrm{~km} \mathrm{~s}^{-1}$ (at $115 \mathrm{GHz}$ ) and $0.17 \mathrm{~km} \mathrm{~s}^{-1}$ (at $110 \mathrm{GHz}$ and $109 \mathrm{GHz}$ ).

The cloudy weather condition during our observations led to system temperatures reaching $550 \mathrm{~K}$ and $350 \mathrm{~K}$ at $115 \mathrm{GHz}$ and $110 \mathrm{GHz}$, respectively. This resulted in rms noises of $1.7 \mathrm{~K}$ and $1.2 \mathrm{~K}$ in the brightness temperature for ${ }^{12} \mathrm{CO} J=1-0$ and ${ }^{13} \mathrm{CO} J=$ $1-0$, respectively. Such large noise would make relatively weak signals undetectable. However, regions with strong line emission can be validly probed. The velocity information provided by these data convincingly reveal the kinematics of the bubble and molecular conditions in some subregions.

\subsection{Other observations}

Public data from infrared to centimeter surveys was used to analyze the bubble N10 at other wavelengths. The GLIMPSE survey (Benjamin et al. 2003) mapped parts of the inner Galactic plane, with IRAC (Infrared Array Camera; Fazio et al. 2004) on Spitzer Space Telescope. We obtained images of 4.5, 5.8 and 8.0 $\mu \mathrm{m}$ IRAC bands Figure 3. The $24 \mu \mathrm{m}$ image of N10 was obtained from another survey of the inner Galactic plane, MIPSGAL, using the MIPS instrument (Multi- band Imaging Photometer for the Spitzer; Rieke et al. 2004). In the panel at $24 \mu \mathrm{m}$ of Figure 3 we can see that this emission fills the whole area indicated by the red ellipse. This emission, typical of galactic bubbles, is caused by warm dust present in the region of ionized gas.

The existence of an HII region inside the bubble is confirmed by the radio continuum emission at 20 $\mathrm{cm}$ from MAGPIS (Helfand et al. 2006). In order to improve the continuum emission data, MAGPIS combined VLA images with images from a $1.4 \mathrm{GHz}$ survey carried out by Reich et al. (1990) using the Effelsberg 100-m telescope. This emission, due to free-free process, is a good tracer of ionized gas.

N10 was mapped at sub-mm wavelengths with the APEX telescope (Miettinen 2012). The images at $870 \mu \mathrm{m}$ wavelength were obtained with ATLASGAL (APEX Telescope Large Area Survey of the Galaxy), an observing program using the LABOCA (Large Apex BOlometer CAmera instrument Schuller et al. 2009). The $870 \mu \mathrm{m}$ cold dust emission is useful to reveal the presence of dense dark clouds. Two of these clouds, reported by Wienen et al. (2012), are seen bordering the bubble, along the upper and left borders of the bubble. The HII region, which is probably expanding, seems to be interacting with these clouds. We used the MAGPIS website ${ }^{3}$ to obtain the images presented Figure 3 ,

We also used the all-sky Wide-Field Infrared Survey Explorer satellite (WISE; Wright et al. 2010) data to analyze the content of young stellar objects in N10, in order to reveal the regions where star formation took place recently and possible gradients of evolutionary stage.

\footnotetext{
${ }^{3}$ http://third.ucllnl.org/gps
} 


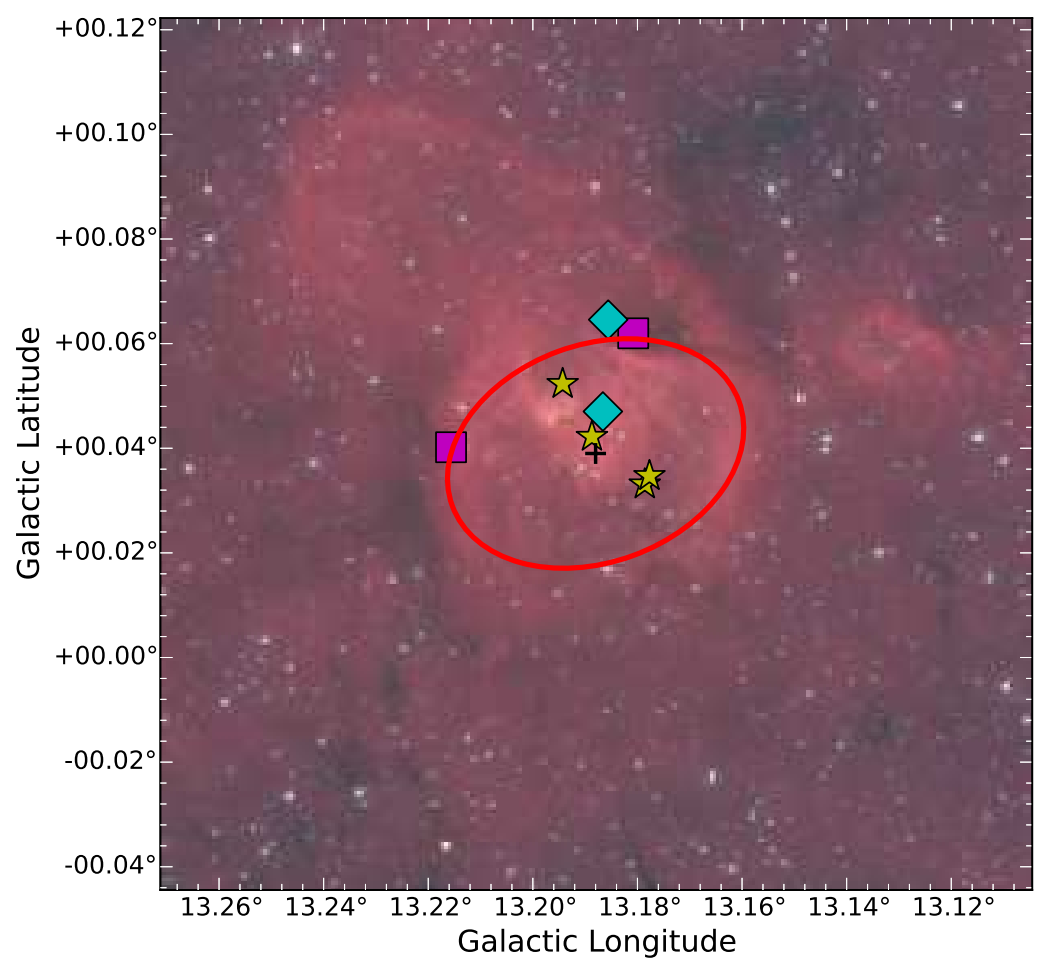

Fig. 2.- Three color composite image of bubble N10 with Spitzer-GLIMPSE $3.6 \mu \mathrm{m}$ (blue), $4.5 \mu \mathrm{m}$ (green) and 8.0 $\mu \mathrm{m}$ (red). The positions of the stars from Watson et al. (2008) are marked as yellow stars; dust condensations identified by Deharveng et al. (2010) are indicated by magenta squares (\#1 at the right side, \#2 at the left side); cyan diamonds represents the position of methanol masers by Szymczak (2000; up) and Pandian (2008; down).

\section{Results}

\subsection{Molecular Emission}

The emission of ${ }^{12} \mathrm{CO},{ }^{13} \mathrm{CO}$ and $\mathrm{C}^{18} \mathrm{O} \mathrm{J}=1-0$ was observed at the same time. Strong emission of ${ }^{12} \mathrm{CO}$ and ${ }^{13} \mathrm{CO}$ was observed; the emission of $\mathrm{C}^{18} \mathrm{O}$ is weak and we do not analyze in this work. The Figure 4 presents the observed spectral lines.

Detected ${ }^{12} \mathrm{CO}$ and ${ }^{13} \mathrm{CO}$ emission allows us to identify three peaks of velocity: at 20, 37 and 52 $\mathrm{km} \mathrm{s}^{-1}$, approximately. The central velocities and line widths were determined by Gaussian fits using the CLASS package (GILDAS software 7 ). In this paper, velocities are referred to the local standard of rest $\left(\mathrm{V}_{L S R}\right)$. Upper panel in Figure 5 displays a channel map of ${ }^{12} \mathrm{CO}$ emission and bottom panel shows the channel map of ${ }^{13} \mathrm{CO}$ emission. The background in

\footnotetext{
${ }^{4}$ http://www.iram.fr/IRAMFR/GILDAS
}

both figures shows $8.0 \mu \mathrm{m}$ emission. We have fitted the channels by increasing the velocity from 45 to 62 $\mathrm{km} \mathrm{s}^{-1}$. There is an strong correlation between ${ }^{12} \mathrm{CO}$ and ${ }^{13} \mathrm{CO}$ emission, specially in the range $48-53 \mathrm{~km}$ $\mathrm{s}^{-1}$.

Broad CO component centered at $20 \mathrm{~km} \mathrm{~s}^{-1}$ has the lower intensity of the three peaks. Component centered at $37 \mathrm{~km} \mathrm{~s}^{-1}$ presents narrower profile than the former, and lower intensity if compared with the component centered at $52 \mathrm{~km} \mathrm{~s}^{-1}$.

The velocities found in the literature for different emission lines associated with $\mathrm{N} 10$ range from 48.5 to $54.1 \mathrm{~km} \mathrm{~s}^{-1}$, as shown in Table 2. This leads us to adopt the component with peak at $52.6 \mathrm{~km} \mathrm{~s}^{-1}$ as the one related to the source.

In our observation, velocities along the emission with peak at $52.6 \mathrm{~km} \mathrm{~s}^{-1}$ range from 48 to $53 \mathrm{~km}$ $\mathrm{s}^{-1}$. In order to verify the correspondence between the physical distribution of molecular gas and the bubble 

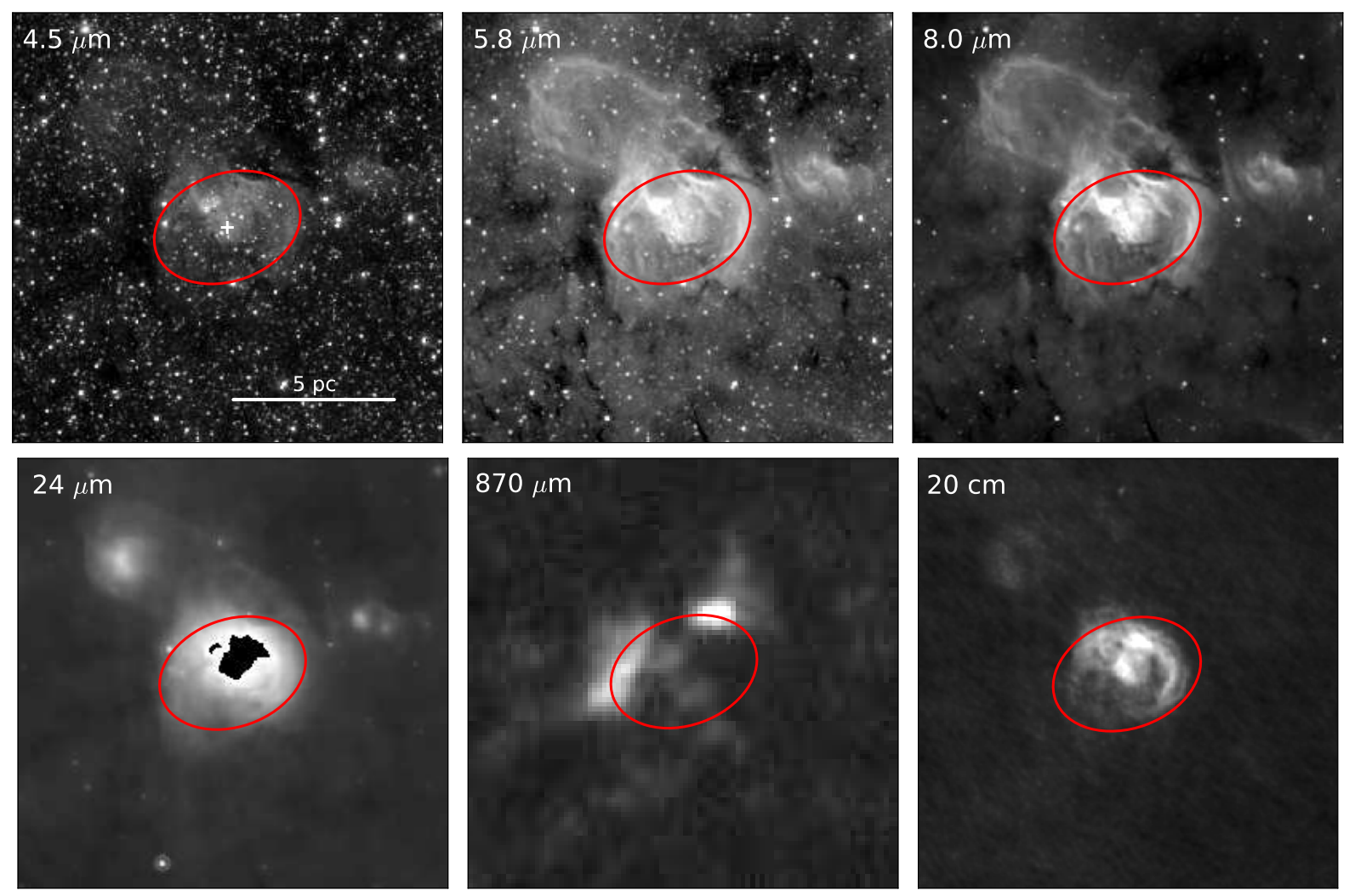

Fig. 3.- Multi-wavelength images of the N10 region. The panels show $4.5 \mu \mathrm{m}, 5.8 \mu \mathrm{m}, 8.0 \mu \mathrm{m}, 24 \mu \mathrm{m}, 870 \mu \mathrm{m}$ and $20 \mathrm{~cm}$ emission towards the target region, from Spitzer-GLIMPSE, Spitzer-MIPSGAL, LABOCA-ATLASGAL and GPS-VLA, respectively. All the images are showing the same region in the sky; in the first panel, white cross marks the center of the HII region; in all images the red ellipse is for reference, as shown in Figure 1.

seen in infrared, ${ }^{12} \mathrm{CO}$ and ${ }^{13} \mathrm{CO}$ contours of narrowvelocity emission were superposed over a Spitzer 8.0 $\mu \mathrm{m}$ image in Figure 6 Spatial distribution of ${ }^{12} \mathrm{CO}$ shows two main structures that seem to be related to the $8.0 \mu \mathrm{m}$ emission, and ${ }^{13} \mathrm{CO}$ presents two denser clumps in the border of the ring morphology of N10, at the same position of ${ }^{12} \mathrm{CO}$ structures.

\subsection{Distribution of gas and dust}

We have studied the bubble N10 through the emission of the $\mathrm{CO}$ and the cold dust, which is useful to reveal the densest and coldest regions of N10. However, it is necessary to explore other tracers.

\section{Ionized gas}

Ionized gas associated with N10 can be traced by VLA $20 \mathrm{~cm}$ emission. The presence of emission at $v=1.5 \mathrm{GHz}$ implies that the HII region in the inner part of the bubble is created by UV photons. Using the Greg/GILDAS software we estimated the 20 $\mathrm{cm}$ total flux $F_{20 \mathrm{~cm}}=1.17 \mathrm{Jy}$ inside the bubble and we calculated the electron density $\left(n_{e}\right)$ according Panagia \& Walmsley (1978):

$$
\begin{aligned}
\frac{\mathrm{n}_{e}}{\mathrm{~cm}^{-3}} & =3.113 \times 10^{2}\left(\frac{\mathrm{T}_{e}}{10^{4} K}\right)^{0.25}\left(\frac{\mathrm{S}_{v}}{\mathrm{~J} y}\right)^{0.5}\left(\frac{\mathrm{D}}{\mathrm{k} p c}\right)^{-0.5} \\
& \times b(v, \mathrm{~T})^{-0.5} \times \theta_{\mathrm{R}}^{-1.5}
\end{aligned}
$$

where $\mathrm{T}_{e}$ in $\mathrm{K}$ is the electron temperature, $\mathrm{S}_{v}$ is the measured total flux density in Jy, D is the distance in $\mathrm{kpc}$ and $\theta_{R}$ is the angular radius in arcmin. The function $\mathrm{b}(v, T)$ is defined as: 

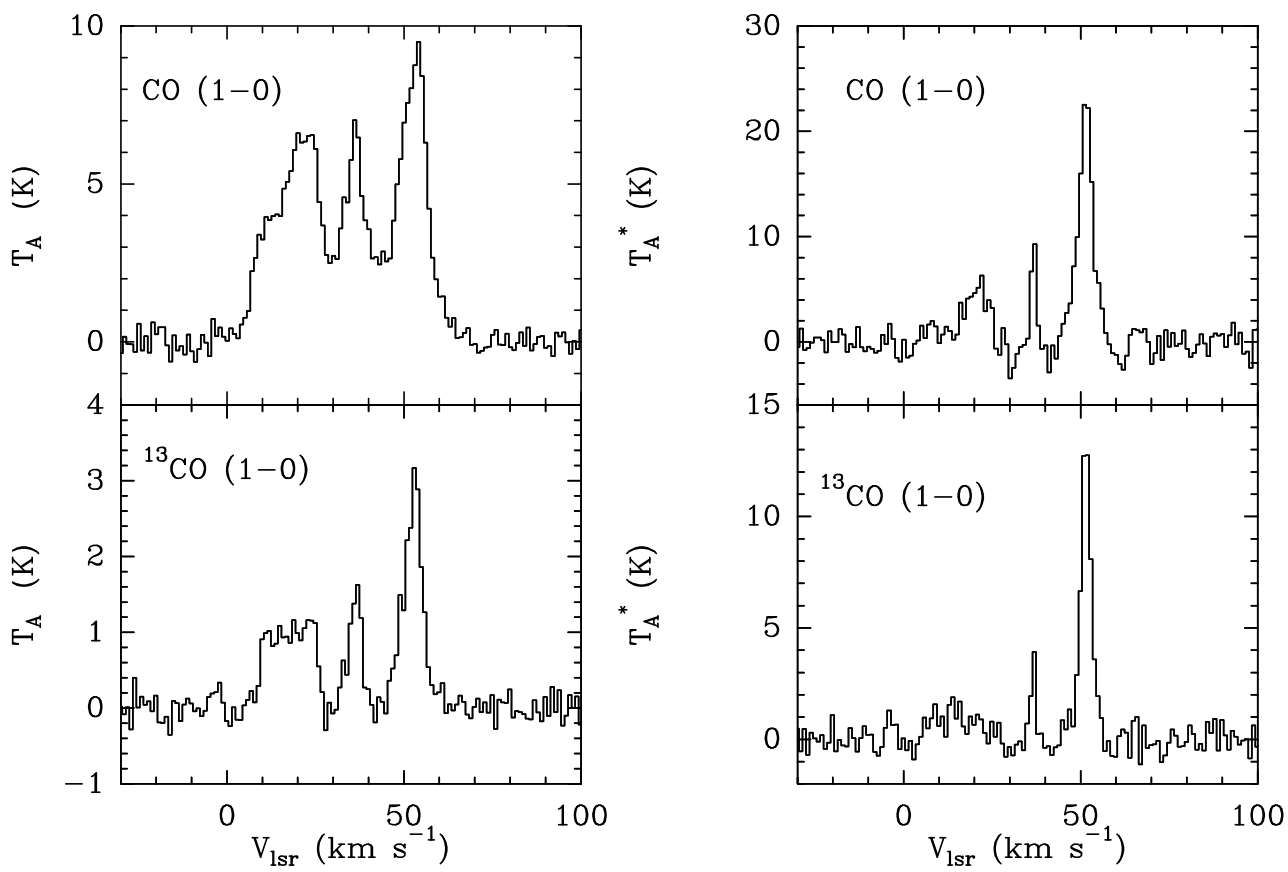

Fig. 4.- Spectral lines. The left column shows the average spectra of $J=1-0$ transition of ${ }^{12} \mathrm{CO}$ in the upper panel and ${ }^{13} \mathrm{CO}$ in the lower panel of the observed region $\left(\Delta l=0.002^{\circ}, \Delta b=0.304^{\circ}\right)$. The right column presents spectra of these two lines in the peak position, at $l=13.21^{\circ}, b=0.037^{\circ}$.

$$
\begin{aligned}
b(v, \mathrm{~T}) & =1+0.3195 \log \left(\frac{\mathrm{T}_{e}}{10^{4} K}\right) \\
& -0.2130 \log \left(\frac{v}{1 G H z}\right) .
\end{aligned}
$$

Assuming $\mathrm{T}_{e}=10^{4} \mathrm{~K}$ for the free-free emission region, the electron density is $\mathrm{n}_{e}=129.71 \mathrm{~cm}^{-3}$.

Figure 7 displays two peaks of radio continuum emission in grayscale and black contours in left panel. In the same figure, right panel shows one of the peaks coinciding with an O-type star.

The number of Lyman continuum photons that are absorbed by the gas in the region HII was calculated using the radio continuum map, following the relation given by Matsakis et al. (1976):

$\mathrm{N}_{u v}=7.5 \times 10^{46}\left(\frac{v}{\mathrm{GHz}}\right)^{0.1}\left(\frac{\mathrm{T}_{e}}{10^{4} K}\right)^{-0.45}\left(\frac{\mathrm{S}_{v}}{\mathrm{Jy}}\right)\left(\frac{\mathrm{D}}{\mathrm{k} p c}\right)^{2}$

We estimate $\mathrm{N}_{u v}=1.86 \times 10^{49}$ ionizing photons $\mathrm{s}^{-1}$ in Lyman continuum, equivalent to a single star type $\mathrm{O}$ (Watson et al. 2008). Considering a model of $\mathrm{H}$ II region in expansion the neutral material accumulates between the ionization front and the shock front (Deharveng et al. 2010), the ionized gas is surrounded by a shell of dense, neutral material hosting PAHs, the main responsible of $8.0 \mu \mathrm{m}$ emission in infrared wavelengths. Therefore ionized gas appears confined by the bubble shell exhibited by $8.0 \mu \mathrm{m}$ emission in red color.

\section{Cold and warm dust}

In Figure 3 it has been displayed the set of emission maps at 4.5, 5.8, 8.0 and $24 \mu \mathrm{m}$. These emissions, corresponding to warm dust, allows to identify clearly the expanding HII region around the bubble N10. The upper panels, maps at $4.5,5.8$ and $8.0 \mu \mathrm{m}$, reveals the arc-shaped boarder of N10.

The thermal emission from cold dust is responsible for mainly continuum $870 \mu \mathrm{m}$ distribution towards $\mathrm{N} 10$, while the emission at $8.0 \mu \mathrm{m}$ originates by polyciclic aromatic hydrocarbons (PAHs) excited by UV photons. Figure 8 displays the emission at $870 \mu \mathrm{m}$ in grayscale and contours for N10 (left panel), and the 

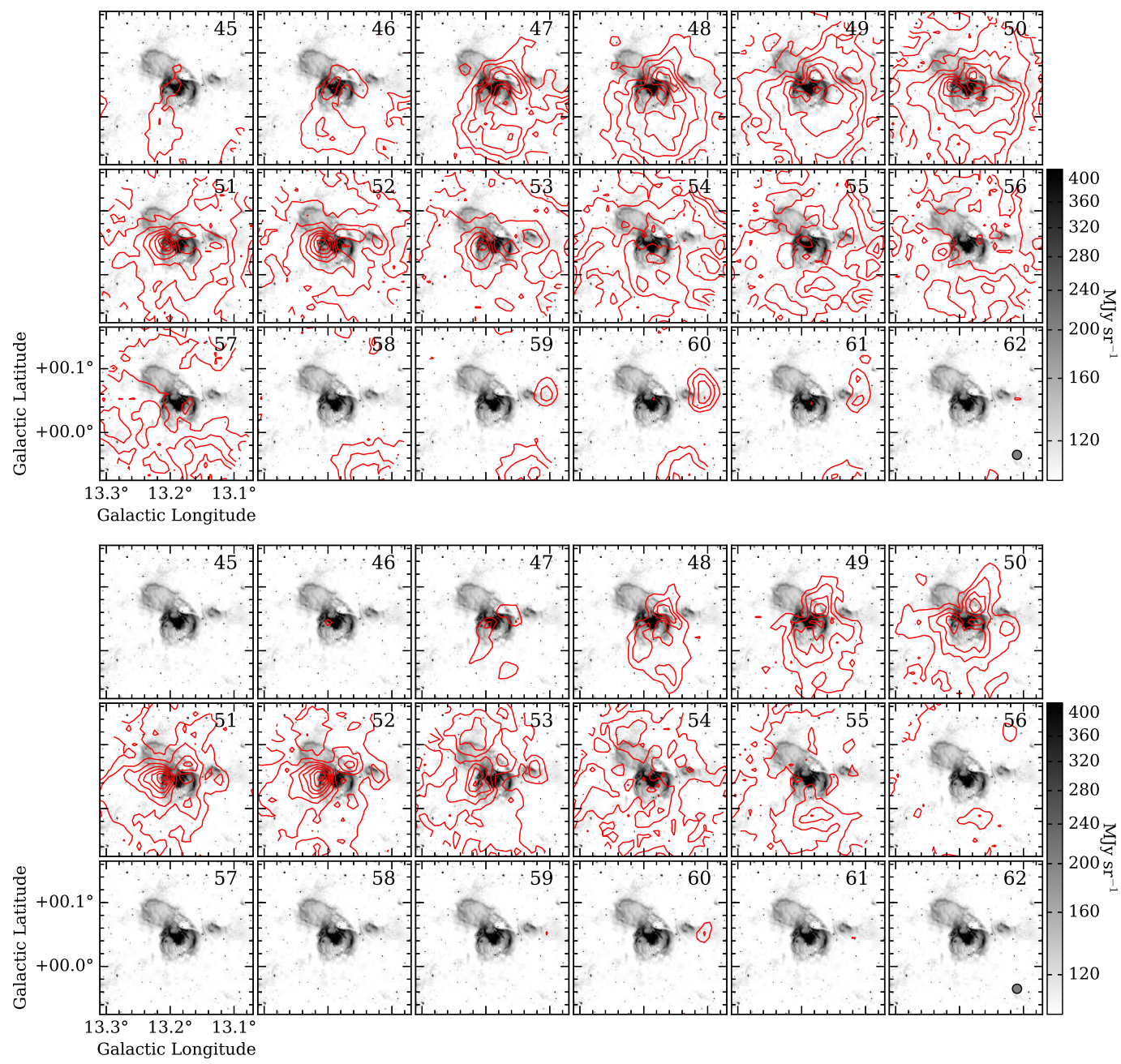

Fig. 5.- Upper panel: Channel maps of ${ }^{12} \mathrm{CO} \mathrm{J}=1-0$ emission in contours superimposed on the Spitzer $8 \mu \mathrm{m}$ image. The contours start from $5 \sigma$ with steps of $3 \sigma\left(1 \sigma=0.90 \mathrm{~K} \mathrm{~km} \mathrm{~s}^{-1}\right)$. The main velocity in $\mathrm{km} \mathrm{s}^{-1}$ is indicated in the left-upper corner of each panel. The small circle represents the beam size of ${ }^{12} \mathrm{CO}$ observations. Lower panel: The same, for ${ }^{13} \mathrm{CO} \mathrm{J}=1-0$ emission. The contours start from $5 \sigma$ increasing with a step of $3 \sigma\left(1 \sigma=0.55 \mathrm{~K} \mathrm{~km} \mathrm{~s}^{-1}\right)$. The scale bar shows the $8 \mu$ flux intensity on a logarithmic scale and the small circle represents the beam size of ${ }^{13} \mathrm{CO}$ observations. 
TABLE 2

Velocities DERIVED TOWARDS THE BUBbLE N10.

\begin{tabular}{ccc}
\hline \hline Velocity & Method & Reference \\
\hline $54.1 \mathrm{~km} \mathrm{~s}^{-1}$ & H II region, radio recombination line & 1 \\
$48.5 \mathrm{~km} \mathrm{~s}^{-1}$ & $6.7 \mathrm{GHz}$ methanol maser emission & 2 \\
$54.1 \mathrm{~km} \mathrm{~s}^{-1}$ & HI absorption line & 3 \\
$50.2 \mathrm{~km} \mathrm{~s}^{-1}$ & CO line emission & 4 \\
$48.5 \mathrm{~km} \mathrm{~s}^{-1}$ & $\mathrm{NH}_{3}$ inversion line (from 870 $\mu$ m data) & 5 \\
$54.1 \mathrm{~km} \mathrm{~s}^{-1}$ & mid-infrared from WISE HII region & 6 \\
\hline
\end{tabular}

References. - (1) Lockman 1989; (2) Szymczak et al. 2000; (3) Pandian et al. 2008; (4) Beaumont \& Williams 2010; (5) Wienen et al. 2012; (6) Anderson et al. 2014.
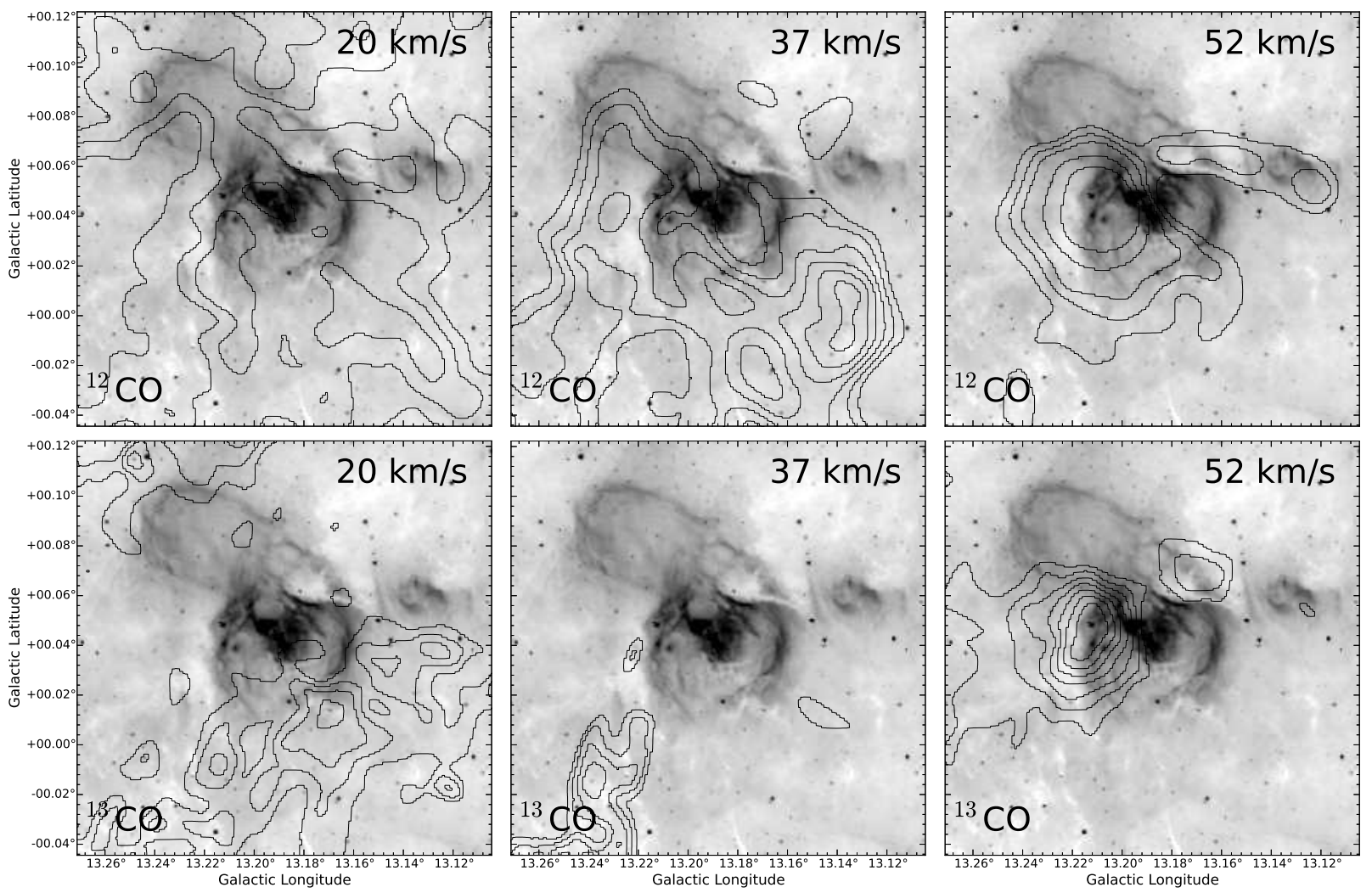

Fig. 6.- The upper panels show contours of ${ }^{12} \mathrm{CO}$ emission distribution centered at $20 \mathrm{~km} \mathrm{~s}^{-1}$ from 6 to 13 -K in steps of $1-\mathrm{K}$ (left); at $37 \mathrm{~km} \mathrm{~s}^{-1}$ in steps of $1-\mathrm{K}$, from 8 to $14-\mathrm{K}$ (middle); at $52 \mathrm{~km} \mathrm{~s}^{-1}$ from 5 to $12-\mathrm{K}$, in steps of $1-\mathrm{K}$ (right). The lower panels display contours of ${ }^{13} \mathrm{CO}$ emission distribution centered at at $20 \mathrm{~km} \mathrm{~s}^{-1}$ from 1 to $3-\mathrm{K}$ in steps of $0.5-\mathrm{K}$ (left); at $37 \mathrm{~km} \mathrm{~s}^{-1}$ in steps of $1-\mathrm{K}$, from 5 to $10-\mathrm{K}$ (middle); at $52 \mathrm{~km} \mathrm{~s}^{-1}$ from 10 to $21-\mathrm{K}$, in steps of $1-\mathrm{K}$ (right). All panels exhibit $8.0 \mu \mathrm{m}$ image in background. 

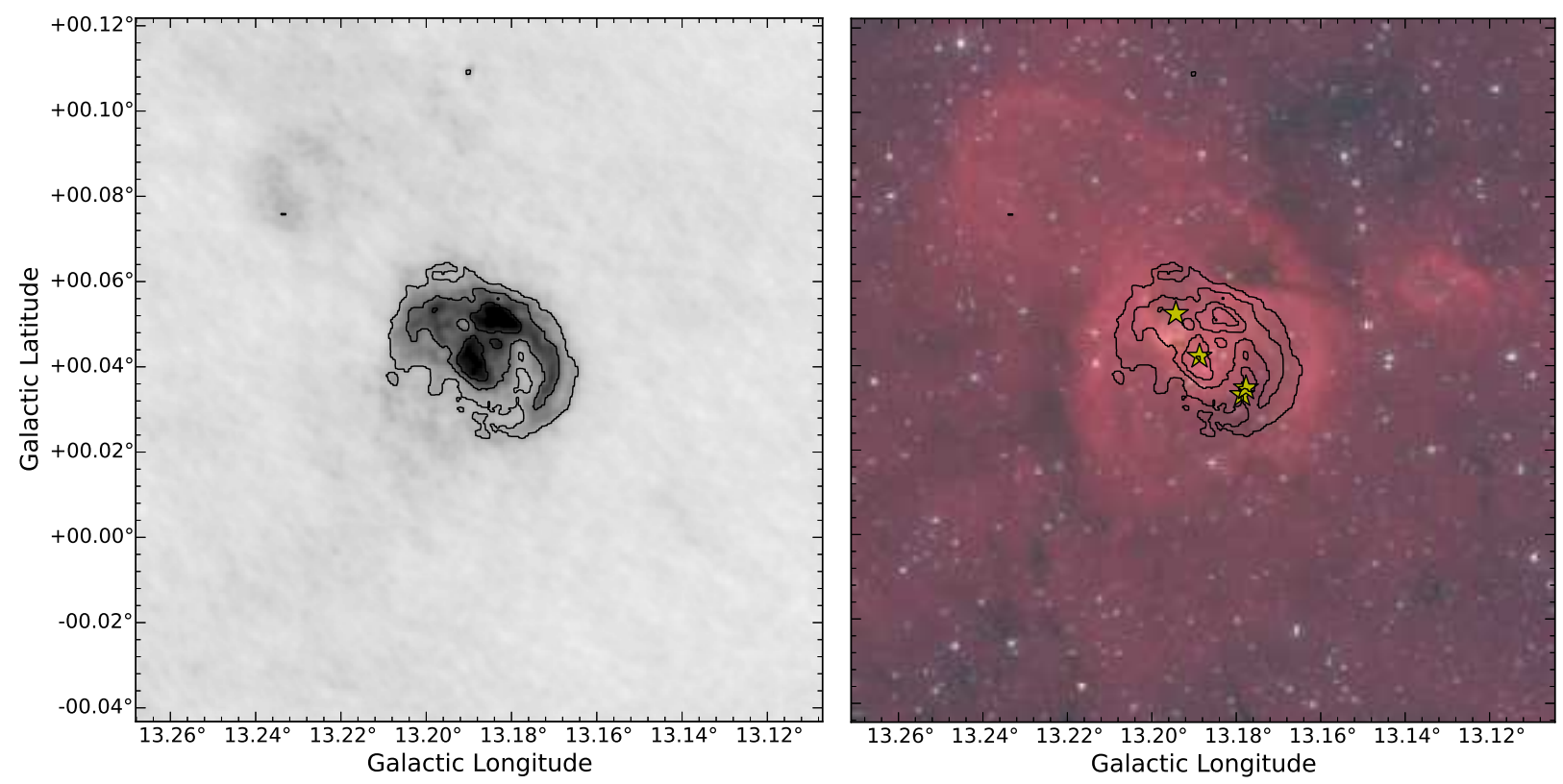

Fig. 7.-Left panel: VLA $20 \mathrm{~cm}(1.4 \mathrm{GHz})$ intensity in grayscale, from 0 to $20 \mathrm{mJy} \mathrm{beam}^{-1}$, with contours start from

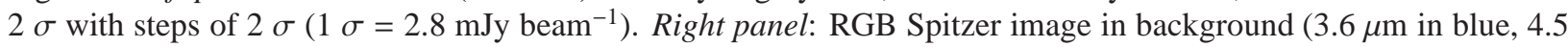
$\mu \mathrm{m}$ in green and $8.0 \mu \mathrm{m}$ in red). Yellow stars marks the position of the candidates ionizing stars.

same contours of the cold dust emission are superimposed on an Spitzer $8.0 \mu \mathrm{m}$ image (right panel).

The two $870 \mu \mathrm{m}$ clumps coincide with ${ }^{13} \mathrm{CO}$ molecular condensations detected at the peak velocity of 52 $\mathrm{km} \mathrm{s}^{-1}$ shown in upper right panel Figure 6 According Dewangan et al. (2015) the coincidence of distribution of the molecular gas, PAH and cold dust emission are an evidence of star-forming material around the bubble. We calculated the physical parameters for the denser condensation in the right of the Figure 8. We here consider that the $870 \mu \mathrm{m}$ radiation originates from the thermal radiation from dust grains. The total mass (dust and gas) in grams can be estimated following relation by Hildebrand (1983):

$$
M_{t o t}=100 \frac{\mathrm{S}_{870 \mu m} D^{2}}{\kappa_{870 \mu m} B_{870 \mu m}\left(\mathrm{~T}_{\text {dust }}\right)},
$$

where $S_{870 \mu m}$ is the flux density of $870 \mu \mathrm{m}$ emission in Jy, $\kappa_{870 \mu m}$ is the dust opacity per unity mass at 870 $\mu \mathrm{m}$ and $B_{870 \mu m}$ is the Planck function for a given dust temperature $\mathrm{T}_{\text {dust }}$ in Jy. We assumed $\mathrm{T}_{\text {dust }}=20 \mathrm{~K}$ and $\kappa_{870 \mu m}=1.8 \mathrm{~cm}^{2} \mathrm{~g}^{-1}$. We assumed a gas-to-dust ratio of 100 (Mathis et al. 1977; Draine \& Anderson 1985).
We can calculate the $\mathrm{H}_{2}$ column density $\mathrm{N}\left(\mathrm{H}_{2}\right)$ using the following formula, by Deharveng et al. (2010):

$$
\mathrm{N}\left(H_{2}\right)=\frac{100 F_{870 \mu m}}{\kappa_{870 \mu m} B_{870 \mu m}\left(\mathrm{~T}_{\text {dust }}\right) 2.8 m_{H} \Omega_{\text {beam }}},
$$

where $\mathrm{N}\left(\mathrm{H}_{2}\right)$ is in $\mathrm{cm}^{-2}$, the surface brightness $\mathrm{F}_{870 \mu \mathrm{m}}$ is in $\mathrm{Jy} \mathrm{beam}^{-1}$, the beam solid angle $\Omega_{\text {beam }}$ is in steradians and the hydrogen mass $\mathrm{m}_{H}$ is in grams.

We calculate the effective radius of the clumps as:

$$
\mathrm{R}_{D}=\left(\frac{\theta_{m a j, D}}{2} \times \frac{\theta_{\min , D}}{2}\right)^{0.5}
$$

where the major and minor deconvolved FWHM of the condensation $\left(\theta_{m a j, D}\right.$ and $\left.\theta_{m i n, D}\right)$ was calculated as:

$\theta_{m a j, D}=\sqrt{\theta_{m a j}^{2}-\theta_{H P B W}^{2}}, \quad \theta_{\min , D}=\sqrt{\theta_{\min }^{2}-\theta_{H P B W}^{2}}$

where $\theta_{m a j}$ and $\theta_{\min }$ are the major and the minor FWHM sizes, respectively, was obtained by using the 
GreG/GILDAS fitting. We found that $\theta_{m a j}=44 \operatorname{arcsec}$ and $\theta_{\min }=32$ arcsec. The half-power beamwidth for $870 \mu \mathrm{m}$ is $\theta_{H P B W}=19.2 \mathrm{arcsec}$. Therefore, $R_{\min , D}=$ $25.6 \operatorname{arcsec}$ and $R_{m a j, D}=39.6 \operatorname{arcsec}(0.6 \mathrm{pc}$ and 0.9 $\mathrm{pc}$, respectively, at a distance of $4.7 \mathrm{kpc}$ ). According to Equation 6 the mean radius deconvolved is $R_{D}=0.36$ $\mathrm{pc}$, at a distance $4.7 \mathrm{kpc}$.

The average volume density for this condensation, assuming a spherical geometry, was calculated according Duronea et al. (2015):

$$
n\left(H_{2}\right)=\frac{\mathrm{M}_{t o t}}{4 / 3 \pi R_{D}^{3} \mu m_{H}},
$$

where $\mu$ is the mean molecular weight and $m_{H}$ is the mass of hydrogen atom. We assumed $\mu=2.33 \mathrm{~g}$ and $\mathrm{m}_{H}=1.67 \times 10^{-24} \mathrm{~g}$.

In short, we found a column density $N\left(H_{2}\right)=6.3 \times$ $10^{22} \mathrm{~cm}^{-2}$, a total mass $\mathrm{M}_{\text {tot }}=240 \mathrm{M}_{\odot}$, the mean radius deconvolved is $\mathrm{R}_{D}=0.36 \mathrm{pc}$ and the average volume density is $\mathrm{n}\left(H_{2}\right)=9.4 \times 10^{4} \mathrm{~cm}^{-3}$.

Warm dust can also be traced in this region by distribution of $24 \mu \mathrm{m}$ emission in grayscale. VLA 20 $\mathrm{cm}$ emission is shown in contours. Warm dust and ionized emissions appears to be quite correlated as Figure 9 displays, as expected for HII regions (e.g. Paladini et al. 2012).

We can notice that the IRAC $24 \mu \mathrm{m}$ emission appears saturated close to the VLA $20 \mathrm{~cm}$ emission. This wavelength band concerns dust and gas ionized emission, which is shown in our results too. From Figure 3 we can see that the saturated region is that both the IRAC and $20 \mathrm{~cm}$ emissions are strong.

In resume, the map at $8.0 \mu \mathrm{m}$ is very useful, since it traces the expanding arc in the upper left part of $\mathrm{N} 10$, this region is associated with ionized gas, excited PAHs and warm dust.

On the other hand, the emission map at $20 \mathrm{~cm}$ exposes the central part of the HII region that is surrounded by the N10 boarder; as would be expected, this region contains dust warmer than the expanding arcs. Furthermore, Figure 7 shows clearly the central part of the HII region.

\subsection{Distance}

Adopting a distance of $4.6 \mathrm{kpc}$ from Pandian et al. (2008) (see also Deharveng et al. 2010), the physical size of the ring is about $4.7 \times 2.5 \mathrm{pc}$. Using this distance, N10 is found to be close to the near extremity of the Galactic bar, a region of intensive star formation (see e.g. the maps of the Galactic arms structure by Hou \& Han 2014).

For this work, using circular Galactic rotation models (e.g. Brand \& Blitz 1993) is possible to compute near and far kinematic distances of the source; we have analyzed the kinematic distance ambiguity (KDA) and results shows the near kinematic one may be more reasonable.

Churchwell et al. (2006) argued that infrared bubbles are more likely located at their near kinematic distances, since objects on the far side of the Galactic disk would be obscured by interstellar extinction and contamination of other structures.

Based on our $\mathrm{CO}$ observations and using the velocity of $52.6 \mathrm{~km} \mathrm{~s}^{-1}$ (see Subsection 4.4), we obtained near and far kinematic distances of $4.7 \mathrm{kpc}$ and 11.3 $\mathrm{kpc}$, respectively. This value is compatible with the near distance estimated by Szymczak et al. (2000) d = $4.4 \mathrm{kpc}$, using the methanol maser emission (see Table 27. Since N10 is located in the inner disk of the Galaxy, we adopt a 10\% uncertainty for the kinematic distance (Yuan et al. 2014), resulting in the value of $4.7 \pm 0.5 \mathrm{kpc}$ for N10.

\subsection{Observed and derived parameters}

In the following discussion we adopt the labels given in Figure 10 to refer to identified condensations. Due to the poor spatial resolution of PMO (the beam size is $\sim 0.9 \mathrm{pc}$ in our observations), the sizes of these molecular condensations could be smaller than $0.9 \mathrm{pc}$.

We used CLASS to calculate the parameters performing Gaussian fits to the average spectra obtained for the whole observed region. We obtained the centroid velocity $\left(\mathrm{V}_{L S R}\right)$, the antenna temperature $\left(\mathrm{T}_{A}^{*}\right)$, and the full width at half-maximum $\left(\Delta \mathrm{V}_{F W H M}\right)$. These observed parameters are shown in Table 3 .

In order to understand the evolutionary status of molecular clumps, we derived the physical properties for the two clumps we identified in our $\mathrm{CO}$ observations. The mass of the clumps were estimated by using the Miriad software package (Sault et al. 1995).

The mass of the molecular gas in a clump is calculated from the intensity of the emission line, under the local thermal equilibrium (LTE) assumption. With the radiation transfer equation Garden et al. (1991) we derived the mass of the clumps under LTE condition, as follow: 

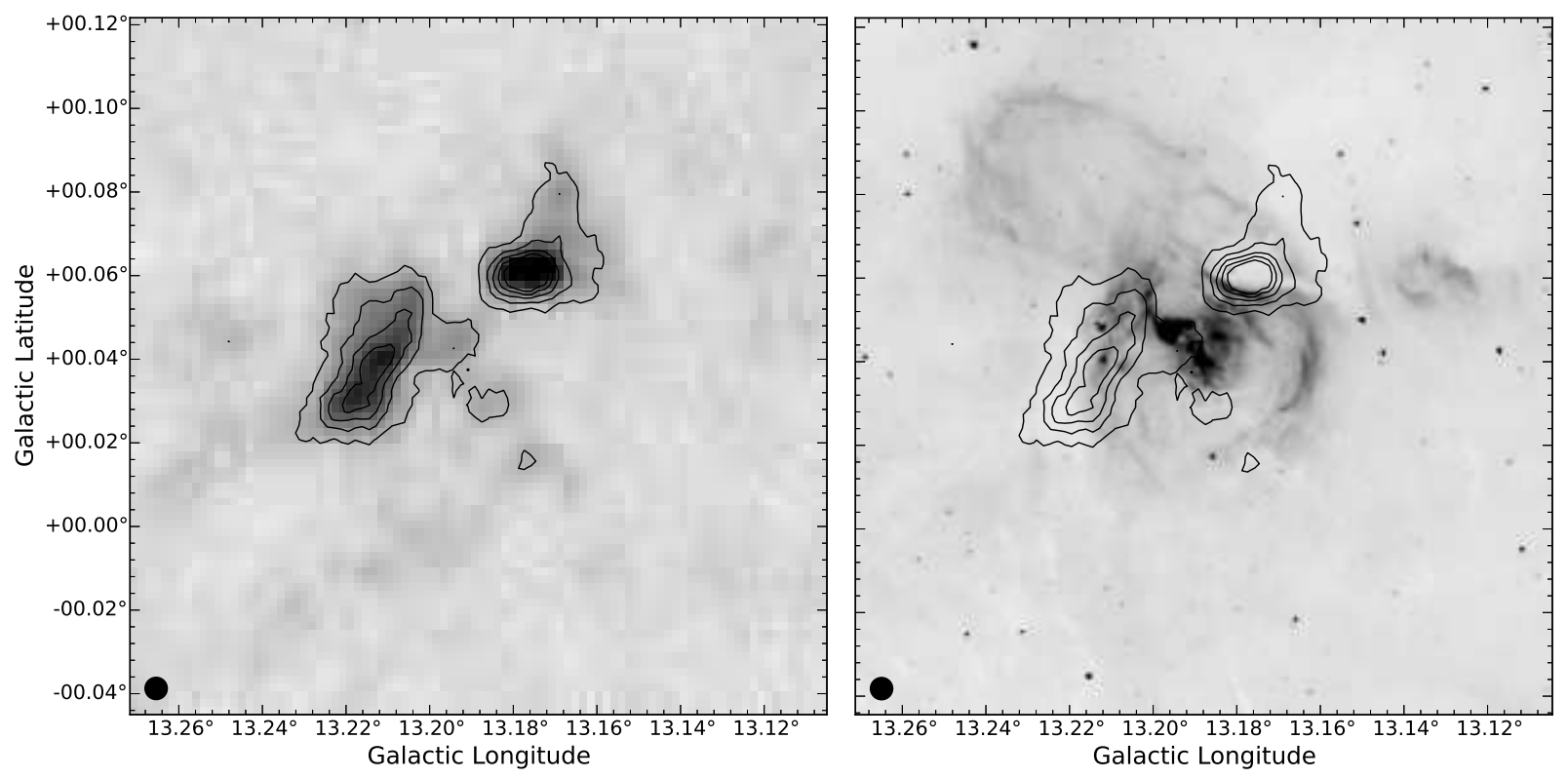

Fig. 8. - Left panel: LABOCA $870 \mu \mathrm{m}$ emission showing the distribution of cold dust in grayscale, from 0 to $5 \mathrm{Jy}$ beam $^{-1}$, with contours starting from $1 \sigma$ to $5 \sigma$, in steps of $1 \sigma\left(1 \sigma=0.5 \mathrm{Jy}_{\text {beam }}{ }^{-1}\right)$. Right panel: Spitzer $8.0 \mu \mathrm{m}$ image in background, overlaid with the same $870 \mu \mathrm{m}$ contours of left figure. In both figures the black dot represents the beam size.

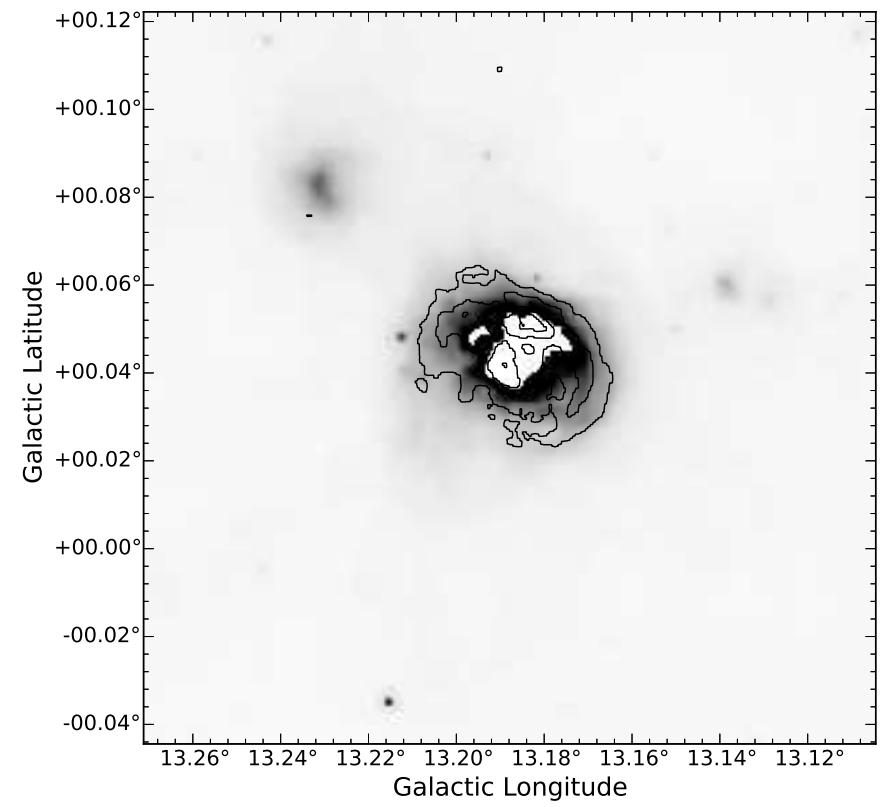

Fig. 9.- $24 \mu \mathrm{m}$ distribution in grayscale from 0 to $2280 \mathrm{MJy} \mathrm{sr}^{-1}$ with the same $20 \mathrm{~cm}$ contours of left panel in Figure 7 


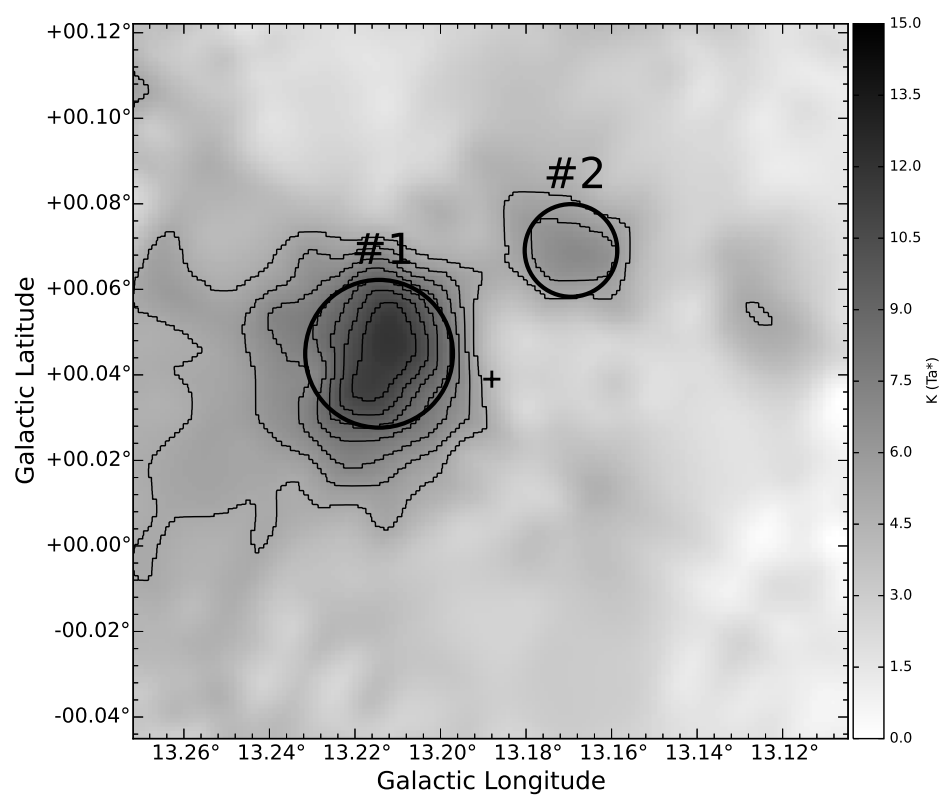

Fig. 10.- Region of the clumps identified in ${ }^{13} \mathrm{CO}$ intensity centered at $52 \mathrm{~km} \mathrm{~s}^{-1}$, in grayscale. Clump \#1 and Clump \#2 seem to be physically associated with bubble N10. Black contours show also ${ }^{13} \mathrm{CO}$ emission from 5 to $11-\mathrm{K}$, in steps of 1-K. The black cross indicates the center of the HII region inside N10.

$$
M_{L T E}=\frac{4}{3} \pi R^{3} m_{H_{2}} \mu_{g} n_{H_{2}}
$$

where $M_{L T E}$ is given in solar masses $M_{\odot}, m_{H_{2}}$ is the mass of hydrogen molecule, $\mu_{g}=1.36$ is the mean atomic weight of the gas and $n_{H_{2}}=N / 2 R$ is the volume density. The mean radius of the clump is obtained by the relation $R=\sqrt{b_{m a j} \times b_{\min }} / 2$, where $b_{m a j}$ and $b_{\min }$ are the sizes of the minor and major axes of the ellipse, respectively, obtained using the Miriad software.

We first estimated the value of the column density $\mathrm{H}_{2}$ through the $\mathrm{CO}$ column density. According to Garden et al. (1991) the column density $N$ of a rigid, asymmetric linear molecule, under LTE condition, can be expressed by:

$$
\begin{aligned}
N & =\frac{3 k}{8 \pi^{3} B \mu^{2}} \times \frac{\exp \left[h B J(J+1) / k T_{e x}\right]}{(J+1)} \\
& \times \frac{\left(T_{e x}+h B / 3 k\right)}{\left[1-\exp \left(-h v / k T_{e x}\right]\right.} \times \int \tau \mathrm{d} v
\end{aligned}
$$

where B is the rotational constant, $\mathrm{J}$ is the rotational quantum number of the lower state of the observed transition and $\mu$ is the electric dipole of the molecule. $T_{e x}$ is the excitation temperature and $\tau$ is the optical depth from 48 to $53 \mathrm{~km} \mathrm{~s}^{-1}$.

Since the excitation temperature $\left(T_{e x}\right)$ is measured as a function of brightness temperature $\left(T_{r}\right)$, we estimate (Garden et al. 1991):

$$
\begin{aligned}
T_{r} & =\frac{h v}{k} \times\left[\frac{1}{\exp \left(h v / k T_{e x}\right)-1}-\frac{1}{\exp \left(h v / k T_{b g}\right)-1}\right] \\
& \times[1-\exp (-\tau)] f
\end{aligned}
$$

where $T_{r}$ is the brightness temperature and the temperature of the cosmic background radiation $T_{b g}=2.73$ K. Here we assume a filling factor of $f=1$.

Assuming that $T_{e x}$ is the same for ${ }^{12} \mathrm{CO}$ and for ${ }^{13} \mathrm{CO}$, the optical depth for both lines can be obtained directly comparing the measure of its brightness temperatures $T_{r}$ (Garden et al. 1991):

$$
\frac{T_{r}\left({ }^{12} C O\right)}{T_{r}\left({ }^{13} C O\right)} \approx \frac{1-\exp \left(-\tau_{12}\right)}{1-\exp \left(-\tau_{13}\right)}
$$

We adopted an isotope ratio of $\left[{ }^{12} \mathrm{CO}\right] /\left[{ }^{13} \mathrm{CO}\right]=60$ 
(Deharveng et al. 2008; Wilson \& Rood 1994), implying $\tau_{12} / \tau_{13}=60$, and the canonical $[\mathrm{CO}] /\left[\mathrm{H}_{2}\right]$ abundance ratio of $10^{-4}$.

Thus we can estimate the optical depth from Equation 12 , and then, using it in Equation 11, and knowing the brightness temperature, we can estimate $T_{\text {ex }}$.

Using the estimated value of $T_{e x}$ for the line ${ }^{13} \mathrm{CO}$, we can finally obtain the hydrogen column density $\left(\mathrm{N}_{\mathrm{H}_{2}}\right)$ using the equation 10 . Thus, the intensity of the ${ }^{13} \mathrm{CO}$ line traces the column density of the clumps \#1 and \#2, as listed in Table 4 .

Obtaining the velocity dispersion and the mass under LTE assumption we can estimate the virial condition, by comparing the gas mass $\left(M_{L T E}\right)$ with the virial mass $\left(M_{\text {viral }}\right)$. In a cloud in which the temporal average kinetic energy is equal to half of the temporal average of the potential energy, the system is considered in virial equilibrium. The assumption that a gravitationally bound system is in virial equilibrium is widely used in astrophysics to estimate its mass (Huang 1954). The virial mass $M_{\text {viral }}$ is given by Ungerechts et al. (2000) by the expression:

$$
\frac{M_{\text {virial }}}{M_{\odot}}=2.10 \times 10^{2}\left(\frac{R}{p c}\right)\left(\frac{\Delta V_{F W H M}}{k m s^{-1}}\right)^{2}
$$

where $\mathrm{R}$ is the mean radius of the clump and $\Delta V_{F W H M}$ is the line width of ${ }^{13} \mathrm{CO}$ line.

The Jeans mass $M_{J}$ is the mass is the mass above which a gas cloud will collapse, for a given density and temperature, when the gravitational attraction overcomes the pressure of the gas. It can be calculated according to Stahler \& Palla (2005):

$$
\frac{M_{\text {Jeans }}}{M_{\odot}}=\left(\frac{T}{10 K}\right)^{3 / 2}\left(\frac{n_{H_{2}}}{10^{4} \mathrm{~cm}^{-3}}\right)^{-1 / 2}
$$

The results are presented in Table 4 where the columns $2-7$ list the following clump parameters: mean radius $(R)$, excitation temperature $\left(T_{e x}\right)$, column density $\left(N_{H_{2}}\right)$, volume density $\left(n_{H_{2}}\right)$, gas mass calculated under LTE assumption $\left(M_{L T E}\right)$, virial mass $\left(M_{\text {virial }}\right)$ and Jeans mass $\left(M_{\text {Jeans }}\right)$. We discuss the clump status in Subsection 5.1

\subsection{Identification of YSOs in the field of $\mathbf{N 1 0}$}

The distribution of Young Stellar Objects (YSOs) plays a major role in the interpretation of the dynamics of star forming region. To identify the YSOs present in the field of N10, we adopted the method described by Koenig \& Leisawitz (2014) (hereafter KL), based on the data of the Wide-field Infrared Survey Explorer (WISE; see Wright et al. 2010). In particular we used the AllWISE release (Cutri et al. 20115, Cutri et al. 2013), which combined the data from the cryogenic and post-cryogenic phases of the survey, resulting in a catalog with enhanced sensitivity.

The catalog was accessed through the VIZIER facility of the Strasbourg Data Center. The catalog contains infrared photometric data at 3.6, 4.9, 5.8 and 22 $\mu \mathrm{m}$ wavelengths, hereafter designated as w1, w2, w3 and w4 bands, respectively. In a first step, we selected all the objects situated in the area around N10 that we explored, in the range of Galactic coordinates $13.11^{\circ}<l<13.27^{\circ}$ and $-0.04^{\circ}<b<0.12^{\circ}$.

We found 565 WISE sources in this area. We next filtered this list of sources by applying a serie of quality criteria defined by $\mathrm{KL}$, that they call the uncertainty/signal-to-noise/chi-squared criteria. The purpose of this is to avoid regions in the space of these parameters with relatively high probability of spurious catalog entry. Accordingly, the Class I YSOs are classified as such if their color matches with all the following criteria:

$$
\begin{aligned}
& w 1-w 3>2.0 \\
& w 1-w 2>-0.42 \times(w 2-w 3)+2.2 \\
& w 1-w 2>0.46 \times(w 1-w 3)-0.9 \\
& w 2-w 3<4.5
\end{aligned}
$$

These conditions reflect the divisions in the SED slope $\alpha=d \log \left(\lambda F_{\lambda}\right) / d \log \lambda$.

The Class II objects were also classified according to $\mathrm{KL}$, whose criteria are:

$$
\begin{aligned}
& w 1-w 2>0.25 \\
& w 1-w 2<-0.9 \times(w 2-w 3)+0.25 \\
& w 1-w 2>0.46 \times(w 2-w 3)-0.9 \\
& w 2-w 3<4.5
\end{aligned}
$$

For w3 we kept only the condition of $\mathrm{S} / \mathrm{N}$ larger than 5. It is considered that if a source satisfies the

Cutri \& IPAC/WISE Science Data Center Team (2011) 
TABLE 3

ObSERVED LiNe PARAMETERS OBTAINED OVER THE INTEGRATED AREA DESCRIBED IN FIgURE 4 where $\mathrm{V}_{l s} r$ IS THE CENTROID VELOCITY OF THE MAIN PEAK, THE $\mathrm{T}_{A}^{*}$ IS THE ANTENNA TEMPERATURE AND $\Delta \mathrm{V}_{F W H M}$ IS THE FULL WIDTH AT HALF-MAXIMUM OF LINES.

\begin{tabular}{cccc}
\hline \hline & $\begin{array}{c}\mathrm{V}_{l s r} \\
\left(\mathrm{~km} \mathrm{~s}^{-1}\right)\end{array}$ & $\begin{array}{c}\mathrm{T}_{A}^{*} \\
(\mathrm{~K})\end{array}$ & $\begin{array}{c}\Delta \mathrm{V}_{F W H M} \\
\left(\mathrm{~km} \mathrm{~s}^{-1}\right)\end{array}$ \\
\hline${ }^{12} \mathrm{CO}$ & 52.6 & 8.3 & 9.2 \\
${ }^{13} \mathrm{CO}$ & 52.6 & 2.9 & 6.1 \\
\hline
\end{tabular}

TABLE 4

DERIVED PARAMETERS OF THE CLUMPS OBSERVED IN ${ }^{13} \mathrm{CO}$.

\begin{tabular}{cccccccc}
\hline \hline clump & $\begin{array}{c}\mathrm{R} \\
(\mathrm{pc})\end{array}$ & $\begin{array}{c}\mathrm{T}_{\text {ex }} \\
(\mathrm{K})\end{array}$ & $\begin{array}{c}\mathrm{N}_{H_{2}} \\
\left(10^{22} \mathrm{~cm}^{-2}\right)\end{array}$ & $\begin{array}{c}\mathrm{n}_{H_{2}} \\
\left(10^{3} \mathrm{~cm}^{-3}\right)\end{array}$ & $\begin{array}{c}\mathrm{M}_{\text {LTE }} \\
\left(10^{3} \mathrm{M}_{\odot}\right)\end{array}$ & $\begin{array}{c}\mathrm{M}_{\text {virial }} \\
\left(10^{3} \mathrm{M}_{\odot}\right)\end{array}$ & $\begin{array}{c}\mathrm{M}_{\text {Jeans }} \\
\left(10^{3} \mathrm{M}_{\odot}\right)\end{array}$ \\
\hline$\# 1$ & 1.1 & 16.8 & 4.1 & 4.2 & 2.6 & 9.3 & 7.0 \\
$\# 2$ & 1.2 & 12.9 & 3.3 & 3.9 & 1.5 & 7.8 & 4.7 \\
\hline
\end{tabular}

criteria of being a true source in any one of the bands, it has little probability of being a fake one and will be included in the final list. After this filtering the list of sources reduced to 407 entries.

Next step was to separate the sources into Class I, Class II, Transition Disks and remaining objects. Following KL, the Class I and Class II YSOs are classified as such, based on the w1-w2 versus w2-w3 color-color diagram only. The regions of the diagram that are used to classify the YSOs are defined by a number of frontier lines, shown in Figure 12. The equations of the lines are given by KL (their equations 12 to 20). We found 12 Class I stars and 91 Class II sources. The Transition Disk stars are selected separately by means of the w1-w2 versus w2-w3 color-color diagram as shown in Figure 13. We found 131 transition disk stars. Note that the selection criteria follow an order of priority: a Class I object will remain Class I even if it also satisfies the criterion for Class II, and next the Class II selection prevails over the following selection. This is why we find many Class II objects in the box defining transition disk stars in Figure 13, they were classified Class II in the previous step, on the basis of the different color-color plot.

This selection of Class I objects is robust, since these objects are well separated from the other classes in the color diagrams. Furthermore, we made experi- ments with another classification scheme available in the literature (using Spitzer data, e.g. Gutermuth et al. 2009) and the same Class I objects were retrieved. On the other hand, the distinction between Class II and Transition Disk is a little arbitrary, as we can see some overlap in Figures 12 and 13. In the samples of objects previously known to belong to given classes, used by KL to decide the position of the frontier lines in Figures 12 and 13. one can see a number of transition disk sources in the locus of Class II and vice versa. So, one must consider that the decision to attribute sources to one or the other classes is only valid in a statistical sense, being correct in about $70 \%$ of the cases.

\subsection{SED fitting}

We have compared the position of Class I YSOs, the most embedded young stellar sources, with the molecular distribution and the objects identified from \#1 to \#9 (see Table 6) are more likely to be physically related to N10 molecular structure. We have fitted their spectral energy distribution (SED) by using the online tool developed by Robitaille et al. (2007). Radiation transfer models were fitted to observational data extracted from WISE catalog based on a $\chi^{2}$ test. We selected models for which $\chi^{2}-\chi_{\text {min }}^{2}<3 n$, where $\chi_{\text {min }}^{2}$ is the minimum value and $n$ is the number of input data.

The fitting was performed using fluxes from WISE 
data, distance ranges from 4.23 to $5.17 \mathrm{kpc}$. Interstellar extinction in the direction of N10 was predict to be approximately $10.7 \mathrm{mag}$ according to model $\mathrm{S}$ of Amôres \& Lépine (2005), values adopted were from 9.7 to 11.7 mag. The best fit are shown in Figure 11 Resulting values for model parameters are given in Table 5. We found that Class I YSOs have stellar mass ranging from $\sim 1$ to $\sim 13 \mathrm{M}_{\odot}$, stellar temperature $\sim 4000-20000 \mathrm{~K}$, total luminosity $\sim 3 \times 10^{1}-1 \times 10^{3}$ $\mathrm{L}_{\odot}$, envelope accretion rate $\sim 9 \times 10^{-8}-3 \times 10^{-3} \mathrm{M}_{\odot}$ $\mathrm{yr}^{-1}$, disk mass $\sim 7 \times 10^{-3}-6 \times 10^{-1} \mathrm{M}_{\odot}$ and stellar ages from $\sim 2 \times 10^{3}$ to $\sim 1 \times 10^{6} \mathrm{yr}$. 

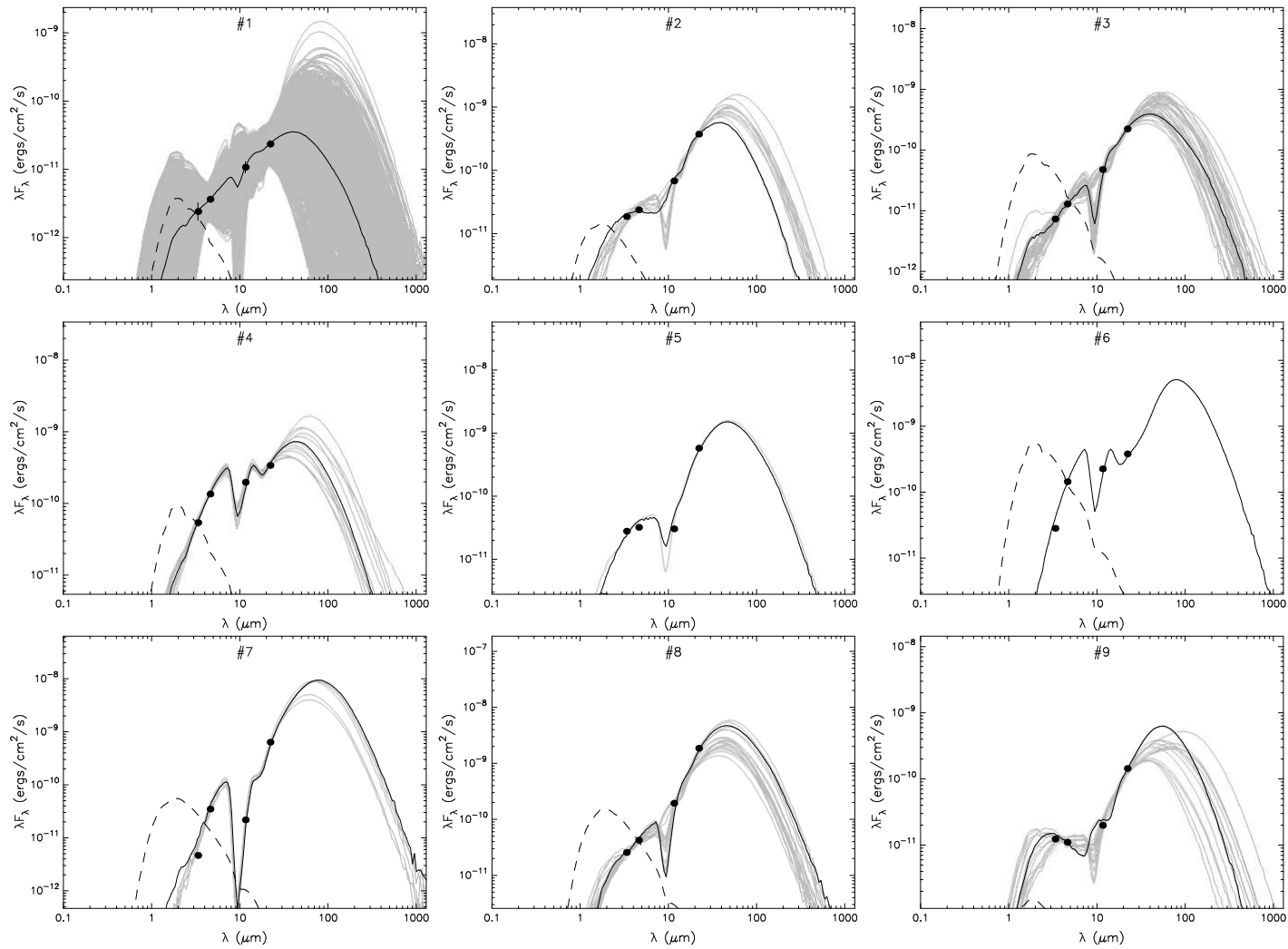

Fig. 11.- The filled circles show the input fluxes. The black line shows the best fit. The dashed line shows the stellar photosphere corresponding to the central source of the best fitting model, as it would loo in the absence of circunstellar dust (but including interstellar extinction). 
TABLE 5

Physical parameters derived from Robitallle et al. (2007) model, for associated Class I objects candidates.

\begin{tabular}{|c|c|c|c|c|c|c|c|c|c|}
\hline Parameters & 1 & 2 & 3 & 4 & 5 & 6 & 7 & 8 & 9 \\
\hline Stellar Mass $\left(\mathrm{M}_{\odot}\right)$ & 1.42 & 6.53 & 7.07 & 6.08 & 7.42 & 9.86 & 12.94 & 12.89 & 6.43 \\
\hline Total Luminosity $\left(\mathrm{L}_{\odot}\right)^{b}$ & $3.11 \times 10^{1}$ & $1.02 \times 10^{3}$ & $5.45 \times 10^{2}$ & $5.96 \times 10^{2}$ & $2.33 \times 10^{3}$ & $2.47 \times 10^{3}$ & $1.13 \times 10^{4}$ & $7.31 \times 10^{3}$ & $1.19 \times 10^{3}$ \\
\hline$\dot{M}_{e n v}\left(\mathrm{M}_{\odot} \mathrm{yr}^{-1}\right)$ & $2.49 \times 10^{-5}$ & $1.20 \times 10^{-5}$ & $4.70 \times 10^{-5}$ & $1.19 \times 10^{-5}$ & $7.60 \times 10^{-5}$ & $1.12 \times 10^{-5}$ & $3.61 \times 10^{-3}$ & $2.25 \times 10^{-4}$ & $8.62 \times 10^{-8}$ \\
\hline Disk Mass $\left(\mathrm{M}_{\odot}\right)^{c}$ & $1.01 \times 10^{-2}$ & $3.05 \times 10^{-2}$ & $3.38 \times 10^{-2}$ & $4.45 \times 10^{-3}$ & $1.46 \times 10^{-3}$ & $7.71 \times 10^{-3}$ & $1.49 \times 10^{-1}$ & $6.09 \times 10^{-1}$ & $5.25 \times 10^{-5}$ \\
\hline
\end{tabular}

${ }^{a}$ Identification from Table 6

${ }^{\mathrm{b}}$ Total system luminosity.

${ }^{\mathrm{c}}$ Envelope and ambient density mass, in solar masses. 
TABLE 6

Young STELLAR OBJects (YSO) CANDIDATES TOWARdS THE N10 REGION.

\begin{tabular}{|c|c|c|c|c|c|c|c|c|}
\hline \multirow{2}{*}{$\begin{array}{c}\text { Candidate } \\
\#\end{array}$} & \multirow{2}{*}{$\begin{array}{l}1 \\
{\left[{ }^{\circ}\right]}\end{array}$} & \multirow{2}{*}{$\begin{array}{l}\mathrm{b} \\
{\left[{ }^{\circ}\right]}\end{array}$} & \multirow[t]{2}{*}{ Identification } & \multicolumn{4}{|c|}{ Fluxes [mag] } & \multirow[t]{2}{*}{ Classification } \\
\hline & & & & {$[3.4]$} & {$[4.6]$} & [12] & [22] & \\
\hline 1 & 13.1141 & 0.0566 & J181354.14-173204.6 & 12.651 & 11.220 & 7.212 & 4.212 & Class I \\
\hline 2 & 13.1385 & 0.0593 & $\mathrm{~J} 181356.48-173043.0$ & 10.428 & 9.181 & 5.204 & 1.246 & Class I \\
\hline 3 & 13.1383 & 0.0566 & $\mathrm{~J} 181357.08-173048.2$ & 11.451 & 9.848 & 5.595 & 1.771 & Class I \\
\hline 4 & 13.2124 & 0.0402 & J181409.60-172722.1 & 9.274 & 7.294 & 4.049 & 1.312 & Class I \\
\hline 5 & 13.2007 & 0.0286 & $\mathrm{~J} 181410.77-172819.3$ & 9.995 & 8.855 & 6.081 & 0.734 & Class I \\
\hline 6 & 13.2126 & 0.0477 & J181407.98-172708.4 & 9.978 & 7.224 & 3.903 & 1.187 & Class I \\
\hline 7 & 13.1818 & 0.0608 & $\mathrm{~J} 181401.37-172823.3$ & 11.937 & 8.766 & 6.442 & 0.629 & Class I \\
\hline 8 & 13.2015 & 0.0554 & $\mathrm{~J} 181404.94-172730.5$ & 10.081 & 8.552 & 4.069 & -0.537 & Class I \\
\hline 9 & 13.2016 & 0.0681 & $\mathrm{~J} 181402.15-172708.2$ & 10.877 & 10.015 & 6.538 & 2.244 & Class I \\
\hline 10 & 13.2155 & -0.0350 & $\mathrm{~J} 181426.60-172921.9$ & 9.291 & 7.367 & 4.237 & 1.531 & Class I \\
\hline 11 & 13.2396 & -0.0057 & $\mathrm{~J} 181423.03-172715.2$ & 12.263 & 11.050 & 8.256 & 5.221 & Class I \\
\hline 12 & 13.1931 & 0.0888 & J181356.55-172659.5 & 12.308 & 10.741 & 6.260 & 3.168 & Class I \\
\hline 13 & 13.1114 & 0.1134 & $\mathrm{~J} 181341.27-173035.2$ & 10.397 & 9.934 & 8.657 & 4.825 & Class II \\
\hline 14 & 13.1135 & -0.0134 & $\mathrm{~J} 181409.56-173407.1$ & 11.620 & 11.198 & 9.670 & 7.655 & Class II \\
\hline 15 & 13.1389 & -0.0279 & $\mathrm{~J} 181415.81-173311.7$ & 11.511 & 11.061 & 9.146 & 6.480 & Class II \\
\hline 16 & 13.1384 & -0.0162 & $\mathrm{~J} 181413.16-173253.3$ & 11.235 & 10.748 & 8.809 & 6.800 & Class II \\
\hline 17 & 13.1147 & -0.0195 & $\mathrm{~J} 181411.06-173413.8$ & 11.343 & 11.046 & 8.968 & 7.287 & Class II \\
\hline 18 & 13.1449 & -0.0193 & J181414.65-173237.9 & 11.031 & 10.757 & 8.852 & 6.340 & Class II \\
\hline 19 & 13.1527 & -0.0295 & J181417.83-173231.1 & 10.529 & 10.021 & 7.684 & 5.341 & Class II \\
\hline 20 & 13.1543 & -0.0234 & J181416.66-173215.4 & 10.758 & 10.158 & 8.237 & 6.232 & Class II \\
\hline 21 & 13.1647 & -0.0310 & J181419.59-173155.6 & 11.511 & 10.498 & 9.022 & 6.565 & Class II \\
\hline 22 & 13.1577 & -0.0263 & $\mathrm{~J} 181417.72-173209.5$ & 10.568 & 10.225 & 8.441 & 5.908 & Class II \\
\hline 23 & 13.1919 & -0.0358 & J181423.94-173037.7 & 10.433 & 10.010 & 8.891 & 6.481 & Class II \\
\hline 24 & 13.1704 & -0.0343 & J181421.01-173143.2 & 10.077 & 9.621 & 8.265 & 5.976 & Class II \\
\hline 25 & 13.2038 & -0.0395 & $\mathrm{~J} 181426.20-173006.5$ & 8.941 & 8.625 & 7.104 & 5.237 & Class II \\
\hline 26 & 13.1103 & 0.0155 & J181402.78-173327.7 & 10.560 & 10.300 & 8.813 & 6.951 & Class II \\
\hline 27 & 13.1370 & -0.0034 & J181410.17-173235.5 & 9.904 & 9.313 & 8.291 & 5.669 & Class II \\
\hline 28 & 13.1452 & -0.0016 & J181410.76-173206.7 & 10.542 & 10.097 & 7.285 & 4.779 & Class II \\
\hline 29 & 13.1257 & -0.0028 & J181408.67-173310.3 & 11.147 & 10.754 & 8.995 & 6.637 & Class II \\
\hline 30 & 13.1362 & -0.0003 & J181409.38-173232.9 & 11.275 & 10.635 & 8.559 & 5.484 & Class II \\
\hline 31 & 13.1269 & 0.0325 & $\mathrm{~J} 181401.03-173205.8$ & 10.224 & 9.903 & 8.315 & 6.522 & Class II \\
\hline 32 & 13.1505 & -0.0153 & J181414.43-173213.6 & 10.636 & 10.131 & 8.669 & 5.761 & Class II \\
\hline 33 & 13.1611 & -0.0034 & J181413.08-173119.4 & 10.832 & 10.136 & 8.904 & 5.365 & Class II \\
\hline 34 & 13.1541 & 0.0016 & J181411.12-173133.1 & 10.988 & 10.541 & 8.519 & 5.942 & Class II \\
\hline 35 & 13.1907 & -0.0196 & J181420.20-173013.7 & 10.467 & 9.685 & 8.212 & 7.706 & Class II \\
\hline 36 & 13.1771 & -0.0256 & J181419.91-173106.9 & 10.485 & 10.058 & 8.892 & 7.684 & Class II \\
\hline 37 & 13.1959 & -0.0330 & J181423.81-173020.5 & 10.700 & 10.302 & 8.892 & 6.814 & Class II \\
\hline 38 & 13.1979 & -0.0191 & $\mathrm{~J} 181420.96-172950.1$ & 11.156 & 10.463 & 9.027 & 6.777 & Class II \\
\hline 39 & 13.1797 & -0.0231 & J181419.68-173054.4 & 10.657 & 10.390 & 8.281 & 6.066 & Class II \\
\hline 40 & 13.2025 & -0.0207 & J181421.88-172938.4 & 10.453 & 9.805 & 8.237 & 5.412 & Class II \\
\hline 41 & 13.1850 & -0.0311 & $\mathrm{~J} 181422.08-173051.7$ & 11.027 & 10.458 & 8.722 & 6.238 & Class II \\
\hline 42 & 13.1763 & -0.0096 & J181416.28-173041.9 & 9.523 & 8.906 & 7.872 & 4.966 & Class II \\
\hline 43 & 13.1529 & 0.0133 & J181408.39-173116.8 & 11.277 & 10.554 & 9.114 & 5.257 & Class II \\
\hline 44 & 13.1778 & 0.0060 & J181413.01-173010.3 & 9.489 & 8.862 & 7.858 & 3.911 & Class II \\
\hline 45 & 13.1627 & 0.0214 & J181407.78-173031.9 & 9.323 & 8.688 & 5.991 & 2.136 & Class II \\
\hline 46 & 13.1732 & 0.0086 & $\mathrm{~J} 181411.88-173020.6$ & 10.801 & 10.366 & 7.612 & 3.212 & Class II \\
\hline 47 & 13.1599 & 0.0238 & $\mathrm{~J} 181406.92-173036.4$ & 9.450 & 8.858 & 6.121 & 2.438 & Class II \\
\hline 48 & 13.1904 & 0.0215 & J181411.09-172903.8 & 11.423 & 10.865 & 9.270 & 2.260 & Class II \\
\hline 49 & 13.1951 & 0.0128 & J181413.58-172903.9 & 9.707 & 8.801 & 6.974 & 2.114 & Class II \\
\hline
\end{tabular}


TABLE 6-Continued

\begin{tabular}{|c|c|c|c|c|c|c|c|c|}
\hline \multirow{2}{*}{$\begin{array}{c}\text { Candidate } \\
\#\end{array}$} & \multirow{2}{*}{$\begin{array}{c}1 \\
{\left[{ }^{\circ}\right]}\end{array}$} & \multirow{2}{*}{$\begin{array}{l}\mathrm{b} \\
{\left[{ }^{\circ}\right]}\end{array}$} & \multirow[t]{2}{*}{ Identification } & \multicolumn{4}{|c|}{ Fluxes [mag] } & \multirow[t]{2}{*}{ Classification } \\
\hline & & & & {$[3.4]$} & {$[4.6]$} & {$[12]$} & [22] & \\
\hline 50 & 13.2076 & 0.0148 & $\mathrm{~J} 181414.64-172821.2$ & 9.698 & 9.104 & 6.214 & 1.898 & Class II \\
\hline 51 & 13.1538 & 0.0598 & $\mathrm{~J} 181358.24-172953.7$ & 9.997 & 9.382 & 8.095 & 4.876 & Class II \\
\hline 52 & 13.1348 & 0.0557 & $\mathrm{~J} 181356.85-173101.0$ & 9.628 & 8.946 & 5.814 & 2.094 & Class II \\
\hline 53 & 13.1236 & 0.0950 & $\mathrm{~J} 181346.80-173028.4$ & 10.652 & 10.249 & 8.394 & 5.963 & Class II \\
\hline 54 & 13.1157 & 0.0930 & $\mathrm{~J} 181346.30-173057.0$ & 11.448 & 11.137 & 9.105 & 7.158 & Class II \\
\hline 55 & 13.1554 & 0.0962 & $\mathrm{~J} 181350.37-172845.8$ & 10.915 & 10.286 & 9.060 & 8.037 & Class II \\
\hline 56 & 13.2163 & 0.0706 & J181403.36-172617.6 & 10.970 & 10.459 & 7.987 & 4.483 & Class II \\
\hline 57 & 13.2151 & 0.0670 & $\mathrm{~J} 181404.00-172627.5$ & 11.001 & 10.383 & 7.583 & 5.756 & Class II \\
\hline 58 & 13.2071 & -0.0271 & J181423.84-172934.7 & 9.319 & 8.922 & 7.300 & 4.959 & Class II \\
\hline 59 & 13.2185 & -0.0141 & $\mathrm{~J} 181422.34-172836.2$ & 10.337 & 9.983 & 7.966 & 4.871 & Class II \\
\hline 60 & 13.2078 & -0.0178 & J181421.88-172916.6 & 9.124 & 8.589 & 7.523 & 4.744 & Class II \\
\hline 61 & 13.2179 & -0.0213 & $\mathrm{~J} 181423.85-172850.7$ & 10.761 & 10.112 & 7.705 & 5.931 & Class II \\
\hline 62 & 13.2204 & 0.0050 & J181418.36-172757.4 & 10.030 & 9.534 & 8.327 & 4.625 & Class II \\
\hline 63 & 13.2143 & 0.0017 & $\mathrm{~J} 181418.35-172822.5$ & 10.872 & 10.285 & 8.820 & 4.903 & Class II \\
\hline 64 & 13.2536 & -0.0052 & $\mathrm{~J} 181424.60-172629.9$ & 10.132 & 9.667 & 7.969 & 5.408 & Class II \\
\hline 65 & 13.2532 & 0.0007 & $\mathrm{~J} 181423.25-172621.3$ & 11.055 & 10.557 & 8.166 & 5.727 & Class II \\
\hline 66 & 13.2621 & 0.0030 & J181423.79-172549.1 & 11.615 & 10.736 & 9.077 & 5.965 & Class II \\
\hline 67 & 13.2553 & -0.0207 & $\mathrm{~J} 181428.22-172651.4$ & 9.615 & 9.146 & 7.810 & 5.657 & Class II \\
\hline 68 & 13.2630 & -0.0075 & $\mathrm{~J} 181426.23-172604.1$ & 11.537 & 10.949 & 9.479 & 5.131 & Class II \\
\hline 69 & 13.2440 & -0.0054 & $\mathrm{~J} 181423.50-172700.7$ & 10.056 & 9.031 & 7.095 & 4.135 & Class II \\
\hline 70 & 13.2507 & -0.0041 & $\mathrm{~J} 181424.00-172637.2$ & 10.300 & 9.913 & 7.819 & 5.102 & Class II \\
\hline 71 & 13.2489 & 0.0039 & $\mathrm{~J} 181422.01-172629.3$ & 7.988 & 7.294 & 5.927 & 4.050 & Class II \\
\hline 72 & 13.2484 & -0.0065 & $\mathrm{~J} 181424.27-172648.7$ & 10.165 & 9.770 & 7.610 & 5.207 & Class II \\
\hline 73 & 13.2669 & -0.0343 & $\mathrm{~J} 181432.62-172638.0$ & 10.417 & 9.654 & 8.019 & 7.414 & Class II \\
\hline 74 & 13.2583 & -0.0373 & $\mathrm{~J} 181432.25-172710.3$ & 11.407 & 10.567 & 8.639 & 8.173 & Class II \\
\hline 75 & 13.2301 & 0.0140 & $\mathrm{~J} 181417.54-172711.3$ & 10.361 & 9.642 & 7.410 & 6.239 & Class II \\
\hline 76 & 13.2255 & 0.0156 & J181416.61-172723.1 & 9.430 & 8.754 & 7.473 & 4.366 & Class II \\
\hline 77 & 13.2451 & 0.0341 & J181414.88-172549.3 & 10.995 & 10.254 & 7.405 & 4.293 & Class II \\
\hline 78 & 13.2404 & 0.0107 & J181419.49-172644.6 & 10.908 & 9.870 & 7.559 & 7.685 & Class II \\
\hline 79 & 13.2343 & 0.0419 & $\mathrm{~J} 181411.87-172610.1$ & 9.853 & 9.426 & 7.266 & 4.838 & Class II \\
\hline 80 & 13.2499 & 0.0332 & J181415.66-172535.5 & 10.933 & 10.088 & 7.836 & 4.427 & Class II \\
\hline 81 & 13.2636 & 0.0175 & $\mathrm{~J} 181420.79-172519.2$ & 9.982 & 9.342 & 8.087 & 5.534 & Class II \\
\hline 82 & 13.2574 & 0.0339 & $\mathrm{~J} 181416.42-172510.8$ & 11.900 & 11.196 & 8.401 & 5.181 & Class II \\
\hline 83 & 13.2392 & 0.0430 & $\mathrm{~J} 181412.20-172552.7$ & 10.673 & 10.118 & 8.891 & 5.524 & Class II \\
\hline 84 & 13.2558 & 0.0378 & J181415.36-172509.1 & 11.519 & 11.054 & 8.415 & 5.495 & Class II \\
\hline 85 & 13.1292 & 0.1156 & J181342.93-172935.3 & 10.796 & 10.527 & 8.517 & 6.073 & Class II \\
\hline 86 & 13.1461 & 0.1132 & $\mathrm{~J} 181345.50-172845.9$ & 11.101 & 10.645 & 8.279 & 6.229 & Class II \\
\hline 87 & 13.1350 & 0.1103 & $\mathrm{~J} 181344.81-172926.0$ & 8.488 & 7.950 & 6.759 & 5.174 & Class II \\
\hline 88 & 13.1616 & 0.1054 & J181349.09-172810.6 & 11.137 & 10.402 & 8.815 & 5.834 & Class II \\
\hline 89 & 13.1883 & 0.0892 & $\mathrm{~J} 181355.88-172714.1$ & 7.892 & 7.571 & 6.055 & 3.938 & Class II \\
\hline 90 & 13.1815 & 0.1000 & $\mathrm{~J} 181352.66-172716.9$ & 10.393 & 9.975 & 7.960 & 6.182 & Class II \\
\hline 91 & 13.1945 & 0.1155 & $\mathrm{~J} 181350.82-172609.0$ & 10.328 & 10.050 & 8.788 & 6.638 & Class II \\
\hline 92 & 13.1833 & 0.1066 & J181351.44-172659.9 & 9.333 & 9.015 & 7.827 & 5.732 & Class II \\
\hline 93 & 13.2189 & 0.0964 & $\mathrm{~J} 181357.99-172524.8$ & 9.412 & 8.517 & 6.523 & 2.770 & Class II \\
\hline 94 & 13.2282 & 0.0955 & J181359.30-172456.9 & 10.304 & 9.777 & 6.744 & 3.229 & Class II \\
\hline 95 & 13.2126 & 0.0974 & $\mathrm{~J} 181357.00-172543.1$ & 9.804 & 9.485 & 7.280 & 3.962 & Class II \\
\hline 96 & 13.2128 & 0.1152 & J181353.08-172511.7 & 10.206 & 9.920 & 7.349 & 4.207 & Class II \\
\hline 97 & 13.2164 & 0.1159 & J181353.36-172459.1 & 9.300 & 10.050 & 8.821 & 4.499 & Class II \\
\hline 98 & 13.2433 & 0.0710 & J181406.52-172451.6 & 9.465 & 9.015 & 6.765 & 3.092 & Class II \\
\hline 99 & 13.2482 & 0.0971 & J181401.34-172351.1 & 9.776 & 9.168 & 8.050 & 4.627 & Class II \\
\hline
\end{tabular}


TABle 6-Continued

\begin{tabular}{|c|c|c|c|c|c|c|c|c|}
\hline \multirow{2}{*}{$\begin{array}{c}\text { Candidate } \\
\#\end{array}$} & \multirow{2}{*}{$\begin{array}{c}1 \\
{\left[{ }^{\circ}\right]}\end{array}$} & \multirow{2}{*}{$\begin{array}{l}\mathrm{b} \\
{\left[{ }^{\circ}\right]}\end{array}$} & \multirow[t]{2}{*}{ Identification } & \multicolumn{4}{|c|}{ Fluxes [mag] } & \multirow[t]{2}{*}{ Classification } \\
\hline & & & & {$[3.4]$} & [4.6] & [12] & [22] & \\
\hline 100 & 13.2679 & 0.0722 & J181409.21-172331.5 & 11.125 & 10.261 & 7.874 & 4.907 & Class II \\
\hline 101 & 13.2659 & 0.0771 & J181407.89-172329.7 & 9.496 & 9.243 & 7.583 & 4.824 & Class II \\
\hline 102 & 13.2420 & 0.1051 & J181358.82-172356.7 & 10.622 & 10.187 & 7.297 & 4.686 & Class II \\
\hline 103 & 13.2390 & 0.1185 & J181355.51-172343.3 & 10.482 & 9.921 & 7.358 & 4.132 & Class II \\
\hline 104 & 13.1380 & -0.0254 & J181415.16-173310.3 & 11.555 & 11.301 & 8.303 & 6.554 & Transition Disk \\
\hline 105 & 13.1131 & -0.0255 & $\mathrm{~J} 181412.18-173429.2$ & 9.828 & 9.621 & 8.645 & 7.095 & Transition Disk \\
\hline 106 & 13.1302 & -0.0333 & J181415.95-173348.5 & 9.932 & 9.692 & 8.278 & 6.252 & Transition Disk \\
\hline 107 & 13.1319 & -0.0260 & $\mathrm{~J} 181414.56-173330.7$ & 9.684 & 9.453 & 8.130 & 5.958 & Transition Disk \\
\hline 108 & 13.1630 & -0.0390 & $\mathrm{~J} 181421.17-173214.8$ & 8.383 & 7.905 & 7.722 & 5.794 & Transition Disk \\
\hline 109 & 13.1434 & -0.0357 & J181418.08-173311.0 & 10.852 & 10.078 & 9.131 & 7.067 & Transition Disk \\
\hline 110 & 13.1559 & -0.0304 & $\mathrm{~J} 181418.41-173222.5$ & 8.913 & 8.355 & 7.577 & 5.329 & Transition Disk \\
\hline 111 & 13.1591 & -0.0291 & $\mathrm{~J} 181418.52-173210.1$ & 8.583 & 8.237 & 8.206 & 6.513 & Transition Disk \\
\hline 112 & 13.1685 & -0.0357 & J181421.10-173151.7 & 9.228 & 8.592 & 8.107 & 6.014 & Transition Disk \\
\hline 113 & 13.1178 & -0.0041 & J181408.01-173337.4 & 10.642 & 9.930 & 9.228 & 6.738 & Transition Disk \\
\hline 114 & 13.1414 & -0.0040 & J181410.84-173222.7 & 11.260 & 10.857 & 7.784 & 5.008 & Transition Disk \\
\hline 115 & 13.1429 & -0.0156 & J181413.57-173237.9 & 8.265 & 8.045 & 8.394 & 6.546 & Transition Disk \\
\hline 116 & 13.1471 & 0.0039 & J181409.76-173151.2 & 10.965 & 10.559 & 7.662 & 5.014 & Transition Disk \\
\hline 117 & 13.1338 & 0.0047 & J181408.00-173231.7 & 11.836 & 11.295 & 8.002 & 5.328 & Transition Disk \\
\hline 118 & 13.1148 & 0.0518 & J181355.31-173210.8 & 11.336 & 11.114 & 7.206 & 5.035 & Transition Disk \\
\hline 119 & 13.1511 & 0.0367 & J181403.02-173042.0 & 10.622 & 10.453 & 9.851 & 5.419 & Transition Disk \\
\hline 120 & 13.1361 & 0.0203 & J181404.83-173157.6 & 7.942 & 7.328 & 6.682 & 4.696 & Transition Disk \\
\hline 121 & 13.1737 & -0.0160 & J181417.38-173101.2 & 11.805 & 11.355 & 8.017 & 4.768 & Transition Disk \\
\hline 122 & 13.1491 & -0.0062 & J181412.24-173202.1 & 9.232 & 9.026 & 7.356 & 4.807 & Transition Disk \\
\hline 123 & 13.1801 & -0.0195 & J181418.90-173047.1 & 9.321 & 8.778 & 7.815 & 5.611 & Transition Disk \\
\hline 124 & 13.1889 & -0.0263 & $\mathrm{~J} 181421.47-173031.1$ & 11.226 & 10.936 & 7.854 & 6.182 & Transition Disk \\
\hline 125 & 13.1775 & -0.0162 & J181417.88-173049.7 & 10.607 & 10.353 & 7.602 & 4.404 & Transition Disk \\
\hline 126 & 13.1810 & -0.0299 & $\mathrm{~J} 181421.31-173102.2$ & 12.121 & 11.943 & 8.638 & 5.979 & Transition Disk \\
\hline 127 & 13.1864 & -0.0094 & J181417.43-173009.7 & 10.567 & 10.264 & 9.263 & 6.273 & Transition Disk \\
\hline 128 & 13.1724 & 0.0203 & J181409.19-173002.7 & 11.192 & 10.765 & 5.473 & 1.320 & Transition Disk \\
\hline 129 & 13.1668 & 0.0212 & J181408.31-173019.1 & 10.302 & 9.588 & 5.375 & 0.938 & Transition Disk \\
\hline 130 & 13.1777 & 0.0131 & J181411.42-172958.4 & 10.984 & 10.516 & 9.688 & 3.001 & Transition Disk \\
\hline 131 & 13.1698 & 0.0259 & J181407.64-173001.4 & 9.631 & 9.229 & 5.086 & 0.549 & Transition Disk \\
\hline 132 & 13.1665 & 0.0306 & $\mathrm{~J} 181406.20-173003.7$ & 10.611 & 9.836 & 4.188 & -0.772 & Transition Disk \\
\hline 133 & 13.1861 & 0.0172 & J181411.53-172925.0 & 7.226 & 6.464 & 5.898 & 1.992 & Transition Disk \\
\hline 134 & 13.2099 & 0.0177 & J181414.29-172808.9 & 11.603 & 10.824 & 5.747 & 1.936 & Transition Disk \\
\hline 135 & 13.1981 & 0.0108 & J181414.38-172858.1 & 11.703 & 11.179 & 6.253 & 1.919 & Transition Disk \\
\hline 136 & 13.2055 & 0.0025 & J181417.10-172849.0 & 11.824 & 11.332 & 7.058 & 3.638 & Transition Disk \\
\hline 137 & 13.2126 & 0.0194 & J181414.22-172757.5 & 12.147 & 11.623 & 6.538 & 1.997 & Transition Disk \\
\hline 138 & 13.1858 & 0.0049 & J181414.20-172947.0 & 10.778 & 10.507 & 7.445 & 4.071 & Transition Disk \\
\hline 139 & 13.1303 & 0.0691 & $\mathrm{~J} 181353.35-173052.1$ & 9.467 & 9.299 & 7.496 & 4.097 & Transition Disk \\
\hline 140 & 13.1286 & 0.0610 & J181354.93-173111.4 & 10.033 & 9.571 & 4.676 & 2.042 & Transition Disk \\
\hline 141 & 13.1316 & 0.0521 & J181357.26-173117.1 & 10.701 & 10.087 & 6.685 & 3.236 & Transition Disk \\
\hline 142 & 13.1122 & 0.0773 & J181349.34-173135.1 & 10.111 & 9.883 & 10.262 & 7.892 & Transition Disk \\
\hline 143 & 13.1187 & 0.0564 & J181354.75-173150.5 & 11.499 & 11.298 & 6.807 & 4.052 & Transition Disk \\
\hline 144 & 13.1589 & 0.0387 & J181403.50-173014.0 & 9.631 & 8.931 & 5.122 & 0.934 & Transition Disk \\
\hline 145 & 13.1421 & 0.0526 & J181358.42-173043.0 & 11.134 & 10.699 & 6.780 & 3.402 & Transition Disk \\
\hline 146 & 13.1280 & 0.0967 & J181346.96-173011.5 & 9.944 & 9.647 & 8.528 & 6.409 & Transition Disk \\
\hline 147 & 13.1250 & 0.0795 & J181350.40-173050.9 & 8.393 & 8.130 & 7.594 & 5.493 & Transition Disk \\
\hline 148 & 13.1524 & 0.0761 & J181354.44-172930.1 & 8.655 & 8.281 & 7.398 & 5.147 & Transition Disk \\
\hline
\end{tabular}


TABle 6-Continued

\begin{tabular}{|c|c|c|c|c|c|c|c|c|}
\hline \multirow{2}{*}{$\begin{array}{c}\text { Candidate } \\
\#\end{array}$} & \multirow{2}{*}{$\begin{array}{c}1 \\
{\left[{ }^{\circ}\right]}\end{array}$} & \multirow{2}{*}{$\begin{array}{l}\mathrm{b} \\
{\left[{ }^{\circ}\right]}\end{array}$} & \multirow[t]{2}{*}{ Identification } & \multicolumn{4}{|c|}{ Fluxes [mag] } & \multirow[t]{2}{*}{ Classification } \\
\hline & & & & [3.4] & & {$[12]$} & [22] & \\
\hline 149 & 13.1753 & 0.0488 & J181403.24-172904.5 & 9.535 & 8.937 & 2.135 & -2.611 & Transition Disk \\
\hline 150 & 13.1832 & 0.0570 & J181402.39-172825.6 & 10.112 & 9.869 & 3.703 & -0.608 & Transition Disk \\
\hline 151 & 13.1712 & 0.0418 & J181404.30-172929.5 & 10.602 & 10.088 & 3.017 & -1.599 & Transition Disk \\
\hline 152 & 13.1826 & 0.0528 & J181403.24-172834.6 & 9.907 & 9.260 & 1.655 & -2.432 & Transition Disk \\
\hline 153 & 13.1639 & 0.0376 & $\mathrm{~J} 181404.35-173000.0$ & 10.127 & 9.568 & 3.913 & -0.276 & Transition Disk \\
\hline 154 & 13.1713 & 0.0395 & J181404.81-172933.3 & 11.091 & 10.374 & 3.963 & -0.653 & Transition Disk \\
\hline 155 & 13.2105 & 0.0229 & $\mathrm{~J} 181413.21-172758.0$ & 11.888 & 11.416 & 5.427 & 1.524 & Transition Disk \\
\hline 156 & 13.2038 & 0.0461 & J181407.28-172739.2 & 10.341 & 9.920 & 4.262 & -0.182 & Transition Disk \\
\hline 157 & 13.2089 & 0.0266 & $\mathrm{~J} 181412.20-172756.8$ & 11.358 & 10.652 & 5.704 & 0.822 & Transition Disk \\
\hline 158 & 13.2130 & 0.0355 & $\mathrm{~J} 181410.72-172728.4$ & 10.631 & 10.347 & 4.588 & 1.039 & Transition Disk \\
\hline 159 & 13.2103 & 0.0242 & J181412.89-172756.1 & 11.682 & 10.981 & 5.123 & & Transition Disk \\
\hline 160 & 13.1939 & 0.0384 & $\mathrm{~J} 181407.78-172823.8$ & 10.681 & 9.957 & 2.256 & -2.989 & Transition Disk \\
\hline 161 & 13.1896 & 0.0791 & J181358.26-172727.3 & 10.666 & 10.425 & 6.035 & 3.807 & Transition Disk \\
\hline 162 & 13.1801 & 0.0761 & J181357.79-172802.5 & 9.975 & 9.662 & 6.309 & 3.485 & Transition Disk \\
\hline 163 & 13.1976 & 0.0803 & J181358.98-172659.8 & 11.393 & 10.948 & 7.773 & 3.752 & Transition Disk \\
\hline 164 & 13.1915 & 0.0868 & $\mathrm{~J} 181356.80-172708.0$ & 11.358 & 10.833 & 6.953 & 4.164 & Transition Disk \\
\hline 165 & 13.1984 & 0.0722 & $\mathrm{~J} 181400.85-172711.2$ & 11.740 & 11.317 & & 3.467 & Transition Disk \\
\hline 166 & 13.1974 & 0.0662 & $\mathrm{~J} 181402.06-172$ & & 9.973 & & & Transition Disk \\
\hline 167 & 13.2222 & 0.0750 & $\mathrm{~J} 18140$ & 9.327 & 8.771 & & & Transition Disk \\
\hline 168 & 13.2081 & 0.0561 & $\mathrm{~J} 18140$ & 10.739 & 10.0 & 4.434 & & Transition Disk \\
\hline 169 & 13.2017 & -0.0300 & $\mathrm{~J} 181423.8$ & 11.641 & 11.3 & 7.013 & 4.566 & Transition Disk \\
\hline 170 & 13.2052 & -0.0235 & J181422.82-172934.6 & 11.378 & 11.2 & 7.666 & 5.331 & Transition Disk \\
\hline 171 & 13.2266 & -0.0156 & J181423.66-172813.2 & 11.528 & 11.3 & 8.565 & 5.659 & Transition Disk \\
\hline 172 & 13.2147 & -0.0087 & J181420.69-172839.2 & 11.950 & 11.775 & 8.072 & 5.042 & Transition Disk \\
\hline 173 & 13.2250 & 0.0111 & $\mathrm{~J} 181417.54-17$ & 10.599 & 10.406 & 8.002 & 5.212 & Transition Disk \\
\hline 174 & 13.2556 & -0.0021 & J181424.15-172618.2 & 10.899 & 10.461 & 9.358 & 5.608 & Transition Disk \\
\hline 175 & 13.2439 & 0.0016 & J181421.94-172648.9 & 10.058 & 9.892 & 6.862 & 4.494 & Transition Disk \\
\hline 176 & 13.2406 & 0.0040 & $\mathrm{~J} 181421.00-172655.2$ & 11.241 & 11.043 & 6.649 & 4.894 & Transition Disk \\
\hline 177 & 13.2484 & 0.0094 & $\mathrm{~J} 181420.74-172621.2$ & 11.502 & 11.349 & 7.695 & 5.089 & Transition Disk \\
\hline 178 & 13.2676 & 0.0133 & $\mathrm{~J} 181422.20-172514.1$ & 9.564 & 9.109 & 8.739 & 6.273 & Transition Disk \\
\hline 179 & 13.2318 & 0.0604 & J181407.47-172546.1 & 9.299 & 9.099 & 7.004 & 4.299 & Transition Disk \\
\hline 180 & 13.2297 & 0.0737 & $\mathrm{~J} 181404.30-172529.9$ & 9.594 & 9.317 & 4.814 & 1.510 & Transition Disk \\
\hline 181 & 13.2416 & 0.0659 & J181407.44-172505.7 & 10.003 & 9.658 & 6.606 & 3.980 & Transition Disk \\
\hline 182 & 13 & 0.0719 & J181 & 12.536 & 11. & 5.976 & 1.5 & Transition Disk \\
\hline 183 & 13.2315 & 0.0551 & $\mathrm{~J} 181$ & 10 & 10. & 7.724 & 5.911 & Transition Disk \\
\hline 184 & 13.2344 & 0.0724 & $\mathrm{~J} 181405$. & 12.369 & 11.8 & 5.563 & 1.525 & Transition Disk \\
\hline 185 & 13.2363 & 0.0654 & J181406.92-172523.1 & 10.862 & 10.665 & 6.555 & 3.011 & Transition Disk \\
\hline 186 & 13.1462 & 0.1193 & $\mathrm{~J} 181344.17-172835.2$ & 9.092 & 8.479 & 7.845 & 5.891 & Transition Disk \\
\hline 187 & 13.1188 & 0.1113 & J181342.64-173015.7 & 10.204 & 9.787 & 8.924 & 6.601 & Transition Disk \\
\hline 188 & 13.1502 & 0.1042 & J181347.97-172848.4 & 9.896 & 9.186 & 8.720 & 6.425 & Transition Disk \\
\hline 189 & 13.1501 & 0.1144 & J181345.71-172831.4 & 9.305 & 9.117 & 8.257 & 6.510 & Transition Disk \\
\hline 190 & 13.1924 & 0.0975 & J181354.55-172646.7 & 9.473 & 9.250 & 8.793 & 4.548 & Transition Disk \\
\hline 191 & 13.1979 & 0.1120 & J181352.00-172604.4 & 8.698 & 8.412 & 8.768 & 6.937 & Transition Disk \\
\hline 192 & 13.1957 & 0.0886 & J181356.90-172651.6 & 11.635 & 11.142 & 6.151 & 3.423 & Transition Disk \\
\hline 193 & 13.2292 & 0.0910 & J181400.42-172501.5 & 11.600 & 11.348 & 6.060 & 1.161 & Transition Disk \\
\hline 194 & 13.2239 & 0.0903 & J181359.92-172519.4 & 12.463 & 11.933 & 7.178 & 1.502 & Transition Disk \\
\hline 195 & 13.2226 & 0.1037 & J181356.81-172500.8 & 11.410 & 10.976 & 6.355 & 3.515 & Transition Disk \\
\hline 196 & 13.2203 & 0.1024 & $\mathrm{~J} 181356.83-172510.0$ & 10.970 & 10.501 & 5.847 & 3.271 & Transition Disk \\
\hline 197 & 13.2295 & 0.0835 & J181402.10-172513.4 & 11.661 & 11.143 & 5.267 & 0.385 & Transition Disk \\
\hline 198 & 13.2246 & 0.1026 & J181357.29-172456.0 & 10.644 & 10.153 & 5.588 & 3.409 & Transition Disk \\
\hline
\end{tabular}


TABLE 6-Continued

\begin{tabular}{|c|c|c|c|c|c|c|c|c|}
\hline \multirow{2}{*}{$\begin{array}{c}\text { Candidate } \\
\#\end{array}$} & \multirow{2}{*}{$\begin{array}{c}1 \\
{\left[{ }^{\circ}\right]}\end{array}$} & \multirow{2}{*}{$\begin{array}{l}\mathrm{b} \\
{\left[{ }^{\circ}\right]}\end{array}$} & \multirow[t]{2}{*}{ Identification } & \multicolumn{4}{|c|}{ Fluxes [mag] } & \multirow[t]{2}{*}{ Classification } \\
\hline & & & & {$[3.4]$} & [4.6] & [12] & {$[22]$} & \\
\hline 199 & 13.2298 & 0.0885 & J181401.03-172504.1 & 11.742 & 11.114 & 5.584 & 1.075 & Transition Disk \\
\hline 200 & 13.2252 & 0.0813 & $\mathrm{~J} 181402.07-172530.9$ & 10.578 & 10.153 & 6.911 & 0.759 & Transition Disk \\
\hline 201 & 13.2284 & 0.1016 & $\mathrm{~J} 181357.97-172445.8$ & 12.267 & 11.619 & 5.539 & 3.212 & Transition Disk \\
\hline 202 & 13.2280 & 0.0838 & $\mathrm{~J} 181401.85-172517.7$ & 11.410 & 11.135 & 5.899 & 0.356 & Transition Disk \\
\hline 203 & 13.2127 & 0.0831 & $\mathrm{~J} 181400.16-172607.3$ & 8.735 & 8.405 & 7.955 & 4.510 & Transition Disk \\
\hline 204 & 13.2229 & 0.0911 & $\mathrm{~J} 181359.62-172521.4$ & 11.294 & 10.889 & 7.497 & 2.031 & Transition Disk \\
\hline 205 & 13.2372 & 0.1141 & $\mathrm{~J} 181356.27-172356.5$ & 8.749 & 8.517 & 7.132 & 4.033 & Transition Disk \\
\hline 206 & 13.2136 & 0.1166 & $\mathrm{~J} 181352.87-172507.0$ & 11.056 & 10.724 & 7.227 & 4.170 & Transition Disk \\
\hline 207 & 13.2205 & 0.1102 & $\mathrm{~J} 181355.12-172455.8$ & 10.310 & 10.150 & 7.548 & 4.420 & Transition Disk \\
\hline 208 & 13.2377 & 0.0983 & J181359.81-172422.0 & 12.059 & 11.550 & 6.093 & 3.520 & Transition Disk \\
\hline 209 & 13.2404 & 0.0764 & J181404.99-172451.4 & 9.730 & 9.387 & 4.296 & 1.688 & Transition Disk \\
\hline 210 & 13.2385 & 0.0818 & $\mathrm{~J} 181403.55-172448.1$ & 10.547 & 9.932 & 4.625 & 1.282 & Transition Disk \\
\hline 211 & 13.2315 & 0.0864 & $\mathrm{~J} 181401.70-172502.2$ & 12.504 & 12.291 & 5.677 & 0.618 & Transition Disk \\
\hline 212 & 13.2323 & 0.0929 & $\mathrm{~J} 181400.37-172448.5$ & 11.057 & 10.655 & 6.449 & 2.195 & Transition Disk \\
\hline 213 & 13.2312 & 0.0824 & $\mathrm{~J} 181402.55-172510.2$ & 10.613 & 9.946 & 4.475 & -0.268 & Transition Disk \\
\hline 214 & 13.2292 & 0.0787 & $\mathrm{~J} 181403.12-172522.9$ & 9.237 & 9.014 & 3.736 & 0.416 & Transition Disk \\
\hline 215 & 13.2371 & 0.0936 & $\mathrm{~J} 181400.78-172432.1$ & 10.320 & 9.876 & 5.402 & 2.454 & Transition Disk \\
\hline 216 & 13.2325 & 0.0770 & J181403.89-172515.4 & 10.064 & 9.692 & 5.063 & -0.432 & Transition Disk \\
\hline 217 & 13.2404 & 0.0940 & $\mathrm{~J} 181401.08-172421.0$ & 9.943 & 9.723 & 4.805 & 3.131 & Transition Disk \\
\hline 218 & 13.2435 & 0.0771 & $\mathrm{~J} 181405.21-172440.2$ & 11.152 & 10.697 & 6.127 & 2.319 & Transition Disk \\
\hline 219 & 13.2468 & 0.0740 & $\mathrm{~J} 181406.27-172435.2$ & 11.786 & 11.424 & 7.352 & 4.218 & Transition Disk \\
\hline 220 & 13.2352 & 0.0992 & $\mathrm{~J} 181359.33-172428.5$ & 9.378 & 9.056 & 5.712 & 2.634 & Transition Disk \\
\hline 221 & 13.2516 & 0.0858 & J181404.24-172359.7 & 8.614 & 8.314 & 7.176 & 4.687 & Transition Disk \\
\hline 222 & 13.2427 & 0.0814 & $\mathrm{~J} 181404.14-172435.4$ & 10.319 & 10.027 & 4.899 & 2.521 & Transition Disk \\
\hline 223 & 13.2390 & 0.0928 & J181401.19-172427.4 & 9.106 & 8.818 & 5.859 & 2.362 & Transition Disk \\
\hline 224 & 13.2471 & 0.0781 & $\mathrm{~J} 181405.40-172427.1$ & 10.256 & 10.098 & 6.963 & 3.952 & Transition Disk \\
\hline 225 & 13.2354 & 0.0725 & $\mathrm{~J} 181405.22-172513.9$ & 10.123 & 9.731 & 4.448 & 1.682 & Transition Disk \\
\hline 226 & 13.2422 & 0.0887 & $\mathrm{~J} 181402.49-172424.4$ & 10.677 & 10.362 & 5.643 & 2.381 & Transition Disk \\
\hline 227 & 13.2676 & 0.0821 & $\mathrm{~J} 181407.00-172315.7$ & 10.238 & 10.018 & 7.799 & 5.071 & Transition Disk \\
\hline 228 & 13.2696 & 0.0742 & $\mathrm{~J} 181408.98-172322.8$ & 10.599 & 10.386 & 7.460 & 4.081 & Transition Disk \\
\hline 229 & 13.2668 & 0.0850 & $\mathrm{~J} 181406.25-172313.1$ & 10.240 & 9.574 & 8.588 & 5.451 & Transition Disk \\
\hline 230 & 13.2589 & 0.0796 & J181406.49-172347.4 & 7.302 & 6.825 & 6.675 & 4.609 & Transition Disk \\
\hline 231 & 13.2457 & 0.1019 & $\mathrm{~J} 181400.00-172350.7$ & 9.816 & 9.327 & 10.194 & 5.108 & Transition Disk \\
\hline 232 & 13.2399 & 0.1093 & $\mathrm{~J} 181357.66-172356.3$ & 9.114 & 8.881 & 7.718 & 4.412 & Transition Disk \\
\hline 233 & 13.2487 & 0.1005 & $\mathrm{~J} 181400.65-172343.4$ & 10.996 & 10.360 & 11.104 & 6.363 & Transition Disk \\
\hline 234 & 13.2372 & 0.1034 & J181358.63-172415.1 & 9.272 & 8.958 & 6.258 & 3.162 & Transition Disk \\
\hline
\end{tabular}

Note.-Table 6 is published in its entirety in the electronic edition of the Astrophysical Journal. A portion is shown here for guidance regarding its form and content. 


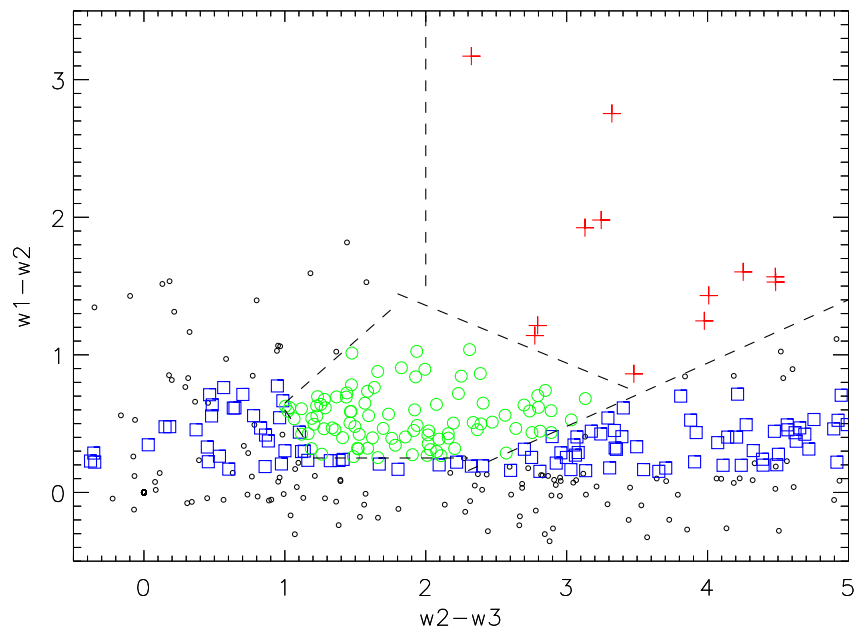

Fig. 12.-Color-color diagram w1-w2 versus w2-w3 of the candidate YSOs around N10. Red crosses: Class I objects; green circles: Class II objects, blue squares: transition disk objects. The dashed lines indicate the limits of the regions according to HL. The transition disks were not defined by means of this diagram. The remaining objects are indicated by black dots.

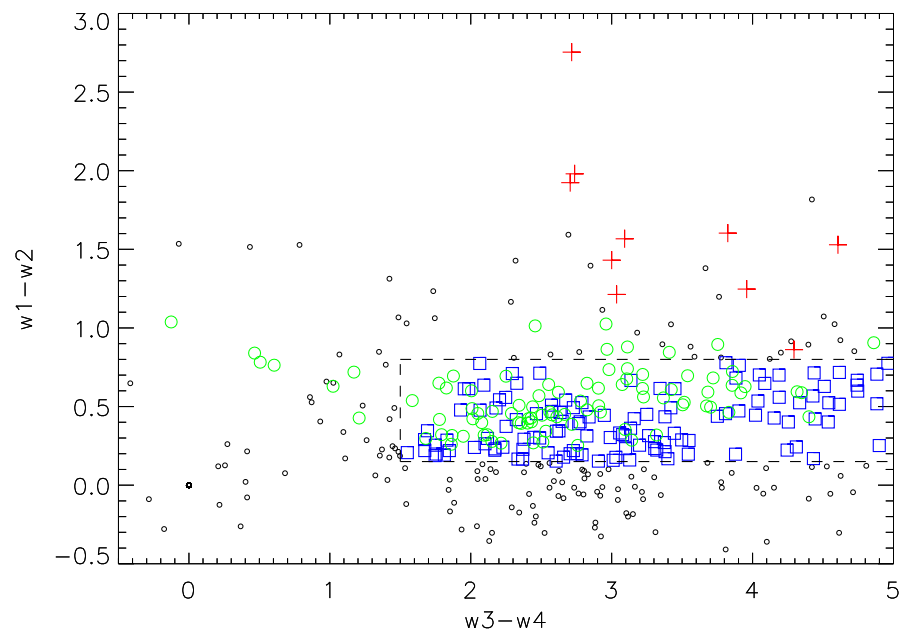

Fig. 13.- Color-color diagram w1-w2 versus w3-w4, like Figure 12. The box used to define transition-disk objects (in blue) also contains many Class II objects (green circles) because these objects were defined to be Class II in a previous step using another color-color diagram (Figure 12). 


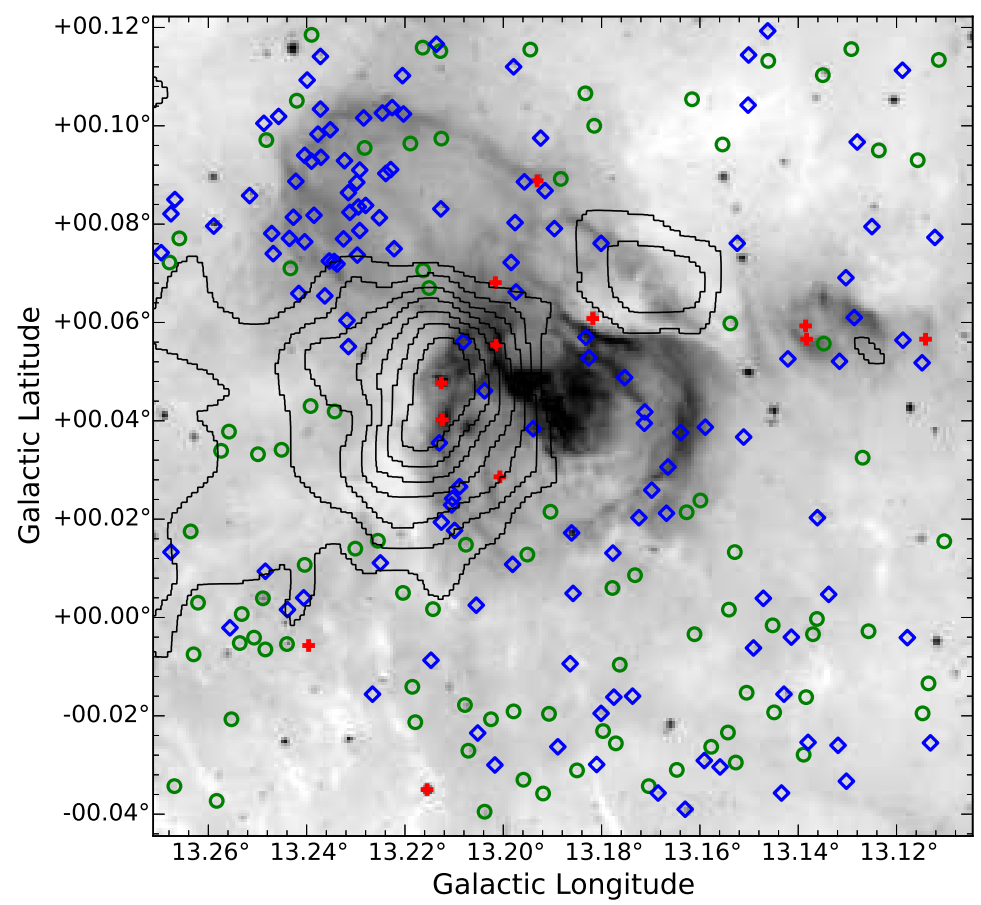

Fig. 14.- Distribution of the identified YSOs. Background is Spitzer $8.0 \mu \mathrm{m}$ image. Red crosses are Class I sources; green circles are class II sources; blue diamonds are "transition disk" sources. Black contours shows the ${ }^{13} \mathrm{CO}$ emission around the bubble. 


\section{Discussion}

In this section we discuss the distribution of molecular material around the bubble N10 and its connection with the star formation history.

\subsection{Surrounding gas of N10}

Channel maps are presented in Figure 5, since ${ }^{12} \mathrm{CO}$ is optically thick, it appears to be spread over a large area, whereas ${ }^{13} \mathrm{CO}$ traces the denser regions, once it is optically thinner. Figure 10 displays two peaks of molecular emission, i.e. two ${ }^{13} \mathrm{CO}$ condensations. Clump \#1 is centered at $l=13.218^{\circ}, b=0.043^{\circ}$ and Clump \#2 is located at $l=13.169^{\circ}, b=0.072^{\circ}$. The two clumps are located precisely on the edge of the ring structure revealed by the $8.0 \mu \mathrm{m}$ emission and highlighted by an ellipse in Figure 2 .

In order to verify the dynamical status of the clumps, we compare the gas mass in LTE and the virial mass calculated in Subsection 4.4 for each clump. The two clumps have greater $\mathrm{M}_{\text {virial }}$ than $\mathrm{M}_{L T E}$, implying that they are gravitationally unbound, indicating currently there is no star in forming (e.g. Yuan et al. 2014; Liu et al. 2016).

\subsection{Other components of $\mathrm{CO}$ emission}

As highlighted in subsection 4.1 $\mathrm{CO}$ components at 20 and $37 \mathrm{~km} \mathrm{~s}^{-1}$ do not seem to be physically related with bubble N10. Which could be the origin of this contribution? It is known $\mathrm{CO}$ emission in galaxies is concentrated in spiral arms (Nieten et al. 2006; Schinnerer et al. 2013). The line of sight towards N10 crosses two spiral arms before reaching the distance $4.7 \mathrm{kpc}$ (see e.g. Figure 10 of Hou \& Han 2014). The velocities of peaks 20 and $37 \mathrm{~km} \mathrm{~s}^{-1}$ correspond to near kinematic distances of 2.4 and $3.7 \mathrm{kpc}$ respectively, representing roughly to the distance of those arms.

It is therefore reasonable to suppose these two velocity peaks are associated with foreground gas situated in distinct spiral arms. Furthermore, emission at these two velocities does not seem to be correlated with geometry of N10; emission appears to be irregularly spread over studied field. If one examines the Figure 4 of the ${ }^{13} \mathrm{CO}(1-0)$ survey of the Galaxy by Lee et al. (2001), selecting the panel corresponding to $b=0.05^{\circ}$, one can see at longitude $13.2^{\circ}$ the presence of ${ }^{13} \mathrm{CO}$ at about 50,35 and $20 \mathrm{~km} \mathrm{~s}^{-1}$. The last two ones are part of elongated structures in the longitude- velocity diagram (extending to higher and lower longitudes) that are usually interpreted as spiral arms. In this region of the diagram, lower velocities correspond to closest arms.

Note that kinematic distances are uncertain at longitudes close to the Galactic center. We have to make use of a ${ }^{13} \mathrm{CO}$ survey because in ${ }^{12} \mathrm{CO}$ longitude-velocity diagrams, the spiral arms are wider in velocity, and are not seen separated.

\subsection{Situation of star formation}

The densest clump in the $870 \mu \mathrm{m}$ emission seems to be a candidate region to form stellar clusters, since it have a total mass of $M_{t o t}=240 \mathrm{M}_{\odot}$ and a mean radius of $R=0.36 \mathrm{pc}$. In accordance with Motte et al. (2003) fragments in the range between 0.09 and 0.56 $\mathrm{pc}$ and masses covering a range from 20 to $3600 \mathrm{M}_{\odot}$ have characteristics of protoclusters.

Elmegreen \& Lada (1977) was the first to propose the scenario of "Collect and Collapse" where the radiation of the massive stars of an HII region creates an ionization front at the interface with the molecular cloud, that drives the propagation of a shock front into the neutral material and which accumulates mass and eventually becomes gravitationally unstable. Other scenarios of triggered star formation have been proposed, like e.g. the "Radiation-Driven Implosion" model, based on the over pressure exerted by the ionized gas, suggested by Lefloch \& Lazareff (1994). While "Collect and Collapse" model takes place in a large spatial size $(\sim 10 \mathrm{pc})$ with a longer timescale (a few Myr), "Radiation-Driven Implosion" takes place in $\sim 1$ pc with a timescale of $0.5 \mathrm{Myr}$.

Although we found evidences for active star formation in N10, we are not sure that the formation of these YSOs were triggered by the "Collect and Collapse" mechanism around the infrared bubble. In order to verify if this process is viable we can apply the analytical model proposed by Whitworth et al. (1994) and compare the fragmentation time scale $t_{\text {frag }}$ with the dynamical age $t_{d y n}$ of the region. The Whitworth et al. (1994) model describes the fragmentation time as:

$$
\mathrm{t}_{\text {frag }}=1.56 c_{s}^{7 / 11} N_{u v}^{-1 / 11} n_{o}^{-5 / 11},
$$

where $c_{S}$ is the isothermal sound speed in the ionized gas in the shocked layer in units of $0.2 \mathrm{~km} \mathrm{~s}^{-1}, N_{u v}$ is the ionizing photon flux in units of $10^{49}$ photons.s ${ }^{-1}$ 
and $n_{o}$ is the initial particle number density of the ambient neutral gas in units of $10^{3} \mathrm{~cm}^{-3}$. Considering $c_{s}=0.2 \mathrm{~km} \mathrm{~s}^{-1}$ (Liu et al. 2012), $N_{u v}=1.86 \times 10^{49}$ photons. $\mathrm{s}^{-1}$ and $n_{o} \sim 10^{3} \mathrm{~cm}^{-3}$ (Ma et al. 2013) we estimated $t_{\text {frag }} \sim 1.5 \times 10^{6} \mathrm{yr}$ for the region. From Ma et al. (2013) $t_{d y n}=9.17 \times 10^{4} \mathrm{yr}$, i.e. the dynamical age is smaller than the fragmentation time scale, which indicates that the region do not support the "Collect and Collapse" mechanism. In this case the "RadiationDriven Implosion" could be considered and further investigated.

The position of YSOs compared with CO distribution indicates that stars are forming inside the molecular clumps. Figure 14 shows the spatial distribution of identified YSOs from the Table 6 In fact the Class I YSOs candidates to be associated to N10 presented in Table 5 have ages smaller than the fragmentation time scale, suggesting a possibility of triggered star formation by pre-existing condensations compressed by the pressure of the ionized gas, as "Radiation-Driven Implosion" scenario proposes.

\subsection{The bubble N11}

Infrared images shows N11, a bubble that seems to be physically connected to N10 and extends about 3 $\mathrm{pc}$ in the up-right direction. Conversely the molecular distribution of $\mathrm{N} 11$ does not suggest a physical connection with $\mathrm{N} 10$, since the emission of ${ }^{13} \mathrm{CO}(1-0)$ between 47 and $53 \mathrm{~km} \mathrm{~s}^{-1}$ is not coincident with 8.0 $\mu$ m emission.

It is probable that this object is a remnant of an HII region, where the lack of $20 \mathrm{~cm}$ emission lead us tho consider there is no more ionized gas inside. It is likely that other energy source has triggered the formation of these YSOs, such as the explosion of a type II supernova.

Class I YSOs do not seem to be superimposed on the bubble N11 and many Class II YSOs can be found towards N11, as we can see in the Figure 14, There is a remarkable concentration of transition disk sources surrounding the top frontier of N11. We consider, in this interpretation, that the concentration of Class II YSOs near the upper frontier of N11 is possibly the result of a past star formation activity related to that bubble.

\subsection{A small bubble to the right of N10: MWP1G013134+000580}

The bubble MWP1G013134+000580, at coordinates $l=13.134^{\circ}$ and $b=0.058^{\circ}$, has size smaller than 2 pc. Interestingly this small bubble should have the about the same distance of $\mathrm{N} 10$, since its $\mathrm{CO}$ emission is contained in the same main velocity peak, clearly seen in channel maps with velocities between 51 and $53 \mathrm{~km} \mathrm{~s}^{-1}$ in Figure 5 .

We found three Class I YSOs in the region covered by $8.0 \mu \mathrm{m}$ emission of MWP1G013134+000580. The age of the small bubble seems to have the same order of N10, as we can infer from the evolutionary stages of the YSOs.

\section{Conclusions}

We have performed a comprehensive study of the infrared bubble N10 using the molecular line emissions of ${ }^{12} \mathrm{CO}(J=1-0)$ and ${ }^{13} \mathrm{CO}(J=1-0)$, mid-infrared Spitzer-GLIMPSE and MIPSGAL images, VLA data of the $20 \mathrm{~cm}$ emission, APEX observations of the continuum $870 \mu \mathrm{m}$ emission and WISE catalog of mid-infrared point sources. The key results are summarized as follows:

1. We observed the $J=1-0$ transition of CO isotopologes at PMO 13.7-m radio telescope. The distribution of the $\mathrm{CO}$ emission showed that the molecular gas around the bubble N10 has velocity $V_{l s r}=52.6 \mathrm{~km} \mathrm{~s}^{-1}$, from which we estimated distance $D=4.7 \pm 0.5 \mathrm{kpc}$. This observations revealed two ${ }^{13} \mathrm{CO}$ clumps with $M_{\text {LTE }} \sim 2 \times 10^{3}$ $\mathrm{M}_{\odot}, M_{\text {virial }} \sim 8.5 \times 10^{3} \mathrm{M}_{\odot}$ and $M_{\text {Jeans }} \sim 6 \times 10^{3}$ $\mathrm{M}_{\odot}$, which means that the clumps implying that they are gravitationally unbound currently.

2. The emission of radio continuum and the presence of $24 \mu \mathrm{m}$ emission suggest ionizing sources inside the bubble. We estimated a total flux of $20 \mathrm{~cm}$ of $F_{20 \mathrm{~cm}}=1.17 \mathrm{Jy}$ and an electron density of $n_{e} \sim 130 \mathrm{~cm}^{-3}$, with a Lyman continuum photon flux of $N_{u v}=1.86 \times 10^{49}$ ionizing photons $\mathrm{s}^{-1}$, equivalent to an $\sim \mathrm{O} 7 \mathrm{~V}$ star (or stars) keeping the gas ionized.

3. Two cold dust clumps were identified towards N10 in LABOCA/APEX images. For the densest clump, we estimated from emission at 870 $\mu \mathrm{m}$ a total mass of $M_{t o t}=240 \mathrm{M}_{\odot}$, a mean radius of $R_{D}=0.36 \mathrm{pc}$, a column density of 
$N\left(H_{2}\right)=6.3 \times 10^{22} \mathrm{~cm}^{-2}$ and an average volume density of $n\left(H_{2}\right)=9.4 \times 10^{4} \mathrm{~cm}^{-3}$, physical characteristics indicating that this condensation is a good candidate of protocluster.

4. We identified 234 YSOs in the whole region: 12 of them classified as Class I, 91 Class II and 131 Transition Disks. We fitted the SED for Class I YSOs candidates identified from \#1 to \#9 and we derived their physical parameters. From the models we found stellar ages ranging from $\sim 10^{3}$ to $10^{6} \mathrm{yr}$. By comparing the estimated dynamical age $\left(t_{d y n}=9.17 \times 10^{4} \mathrm{yr}\right)$ and the fragmentation time scale $\left(t_{\text {frag }} \sim 1.5 \times 10^{6} \mathrm{yr}\right)$ we infer that star formation can be triggered as a consequence of the "Radiation-Driven Implosion" process. Likewise, the age range for the Class I YSOs are below that found for the fragmentation time scale, indicating they were formed before the collect molecular cloud became gravitationally unstable to fragment to form stars.

5. In the Spitzer $8.0 \mu \mathrm{m}$ image the infrared bubble N11 can be seen in the direction of the N10, however one is not physically connected with other. Class II YSOs appears towards N11, suggesting that this could be a remnant of HII region. A third infrared bubble, the small MWP1G013134+000580, appears in the observed field and, interestingly, has $\mathrm{CO}$ emission in the same main velocity as N10 and seems to shelter some evolved YSOs.

We gratefully acknowledge the contribution of Tie Liu, who provided some of the scripts used in this work. We also give our thanks Cristina Cappa and Bertrand Lefloch for the helpful discussion. We thank the anonymous referee for useful comments and suggestions that led to a improved version of the original article. This work is supported by the National Natural Science Foundation of China through grant NSFC 11373009-11433008. J.Y is supported by the National Natural Science Foundation of China through grants of 11503035 and 11573036. EM acknowledges support from the Brazilian agency FAPESP under the grants 2014/22095-6 and 2015/22254-0. We are grateful to the staffs at the Qinghai Station of PMO for their hospitality and assistance during the observations. We thank the Key Laboratory for Radio Astronomy, CAS, for partial support in the operation of the telescope.

\section{REFERENCES}

Amôres, E. B., \& Lépine, J. R. D. 2005, AJ, 130, 659

Anderson, L. D., Bania, T. M., Balser, D. S., et al. 2014, ApJS, 212, 1

Beaumont, C. N., \& Williams, J. P. 2010, ApJ, 709, 791

Becker, R. H., White, R. L., Helfand, D. J., \& Zoonematkermani, S. 1994, ApJS, 91, 347

Benjamin, R. A., Churchwell, E., Babler, B. L., et al. 2003, PASP, 115, 953

Bergin, E. A., \& Tafalla, M. 2007, ARA\&A, 45, 339

Beuther, H., Linz, H., Henning, T., et al. 2011, A\&A, 531, AA26

Blaauw, A. 1991, NATO ASIC Proc. 342: The Physics of Star Formation and Early Stellar Evolution, 125

Brand, J., \& Blitz, L. 1993, A\&A, 275, 67

Bressert, E., Bastian, N., Gutermuth, R., et al. 2010, MNRAS, 409, L54

Cappa, C. E., Rubio, M., Martín, M. C., \& Romero, G. A. 2009, A\&A, 508, 759

Cappa, C. E., Duronea, N., Firpo, V., et al. 2016, A\&A, 585, A30

Churchwell, E., Povich, M. S., Allen, D., et al. 2006, ApJ, 649, 759

Churchwell, E., Watson, D. F., Povich, M. S., et al. 2007, ApJ, 670, 428

Churchwell, E., Babler, B. L., Meade, M. R., et al. 2009, PASP, 121, 213

Condon, J. J., Cotton, W. D., Greisen, E. W., et al. 1998, AJ, 115, 1693

Cutri, R. M., \& IPAC/WISE Science Data Center Team 2011, Bulletin of the American Astronomical Society, 43, \#301.02

Cutri, R. M., et al. 2013, VizieR Online Data Catalog, 2328, 0

Dale, J. E., Bonnell, I. A., Clarke, C. J., \& Bate, M. R. 2005, MNRAS, 358, 291

Dale, J. E., \& Bonnell, I. A. 2008, MNRAS, 391, 2 
Deharveng, L., Lefloch, B., Kurtz, S., et al. 2008, A\&A, 482, 585

Deharveng, L., Schuller, F., Anderson, L. D., et al. 2010, A\&A, 523, A6

Dewangan, L. K., Ojha, D. K., Anandarao, B. G., Ghosh, S. K., \& Chakraborti, S. 2012, ApJ, 756, 151

Deharveng, L., Zavagno, A., Samal, M. R., et al. 2015, A\&A, 582, A1

Dewangan, L. K., \& Ojha, D. K. 2013, MNRAS, 429, 1386

Dewangan, L. K., Ojha, D. K., Grave, J. M. C., \& Mallick, K. K. 2015, MNRAS, 446, 2640

Draine, B. T., \& Anderson, N. 1985, ApJ, 292, 494

Duronea, N. U., Vasquez, J., Gómez, L., et al. 2015, A\&A, 582, A2

Dyson, J. E., \& Williams, D. A. 1980, New York, Halsted Press, 1980. 204 p.,

Elmegreen, B. G., \& Lada, C. J. 1977, ApJ, 214, 725

Fazio, G. G., Hora, J. L., Allen, L. E., et al. 2004, ApJS, 154, 10

Feigelson, E. D., \& Montmerle, T. 1999, ARA\&A, 37, 363

Garden, R. P., Hayashi, M., Hasegawa, T., Gatley, I., \& Kaifu, N. 1991, ApJ, 374, 540

Gutermuth, R. A., Megeath, S. T., Myers, P. C., et al. 2009, ApJS, 184, 18

Helfand, D. J., Becker, R. H., White, R. L., Fallon, A., \& Tuttle, S. 2006, AJ, 131, 2525

Hildebrand, R. H. 1983, QJRAS, 24, 267

Hou, L. G., \& Han, J. L. 2014, A\&A, 569, AA125

Huang, S.-S. 1954, AJ, 59, 137

Ji, W.-G., Zhou, J.-J., Esimbek, J., et al. 2012, A\&A, 544, A39

Kendrew, S., Simpson, R., Bressert, E., et al. 2012, ApJ, 755, 71

Kendrew, S., Zieleniewski, S., Houghton, R. C. W., et al. 2016, MNRAS, 458, 2405
Koenig, X. P., Leisawitz, D. T., Benford, D. J., et al. 2012, ApJ, 744, 130

Koenig, X. P., \& Leisawitz, D. T. 2014, ApJ, 791, 131

Lee, J.-K., Walsh, A. J., Burton, M. G., \& Ashley, M. C. B. 2001, MNRAS, 324, 1102

Lefloch, B., \& Lazareff, B. 1994, A\&A, 289, 559

Lefloch, B., Cernicharo, J., Rodríguez, L. F., et al. 2002, ApJ, 581, 335

Lefloch, B., Cernicharo, J., Cabrit, S., \& Cesarsky, D. 2005, A\&A, 433, 217

Liu, T., Wu, Y., Zhang, H., \& Qin, S.-L. 2012, ApJ, 751,68

Liu, H.-L., Wu, Y., Li, J., et al. 2015, ApJ, 798, 30

Liu, H.-L., Li, J.-Z., Wu, Y., et al. 2016, ApJ, 818, 95

Lockman, F. J. 1989, ApJS, 71, 469

Ma, Y., Zhou, J., Esimbek, J., et al. 2013, Ap\&SS, 345, 297

Mathis, J. S., Rumpl, W., \& Nordsieck, K. H. 1977, ApJ, 217, 425

Matsakis, D. N., Evans, N. J., II, Sato, T., \& Zuckerman, B. 1976, AJ, 81, 172

Minier, V., \& Booth, R. S. 2002, A\&A, 387, 179

Miettinen, O. 2012, A\&A, 542, A101

Motte, F., Schilke, P., \& Lis, D. C. 2003, ApJ, 582, 277

Nieten, C., Neininger, N., Guélin, M., et al. 2006, A\&A, 453, 459

Panagia, N., \& Walmsley, C. M. 1978, A\&A, 70, 411

Pandian, J. D., \& Goldsmith, P. F. 2007, ApJ, 669, 435

Pandian, J. D., Momjian, E., \& Goldsmith, P. F. 2008, A\&A, 486, 191

Paladini, R., Umana, G., Veneziani, M., et al. 2012, ApJ, 760, 149

Pillai, T., Wyrowski, F., Hatchell, J., Gibb, A. G., \& Thompson, M. A. 2007, A\&A, 467, 207

Reich, W., Fuerst, E., Reich, P., \& Reif, K. 1990, A\&AS, 85, 633 
Rieke, G. H., Young, E. T., Engelbracht, C. W., et al. 2004, ApJS, 154, 25

Robitaille, T. P., Whitney, B. A., Indebetouw, R., \& Wood, K. 2007, ApJS, 169, 328

Roman-Duval, J., Jackson, J. M., Heyer, M., et al. 2009, ApJ, 699, 1153

Samal, M. R., Zavagno, A., Deharveng, L., et al. 2014, A\&A, 566, A122

Sault, R. J., Teuben, P. J., \& Wright, M. C. H. 1995, Astronomical Data Analysis Software and Systems IV, 77,433

Schinnerer, E., Meidt, S. E., Pety, J., et al. 2013, ApJ, 779,42

Schuller, F., Menten, K. M., Contreras, Y., et al. 2009, A\&A, 504, 415

Shan, W., Yang, J., Shi, S., et al. 2012, Transactions on Terahertz Science and Technology, 2, 593

Simpson, R. J., Povich, M. S., Kendrew, S., et al. 2012, MNRAS, 424, 2442

Stahler, S. W., \& Palla, F. 2005, The Formation of Stars, by Steven W. Stahler, Francesco Palla, pp. 865. ISBN 3-527-40559-3. Wiley-VCH , January 2005

Szymczak, M., Hrynek, G., \& Kus, A. J. 2000, A\&AS, 143,269

Szymczak, M., Kus, A. J., Hrynek, G., Kěpa, A., \& Pazderski, E. 2002, A\&A, 392, 277

Tackenberg, J., Beuther, H., Henning, T., et al. 2012, A\&A, 540, AA113

Thompson, M. A., Hatchell, J., Walsh, A. J., MacDonald, G. H., \& Millar, T. J. 2006, A\&A, 453, 1003

Thompson, M. A., Urquhart, J. S., Moore, T. J. T., \& Morgan, L. K. 2012, MNRAS, 421, 408

Ungerechts, H., Umbanhowar, P., \& Thaddeus, P. 2000, ApJ, 537, 221

Watson, C., Povich, M. S., Churchwell, E. B., et al. 2008, ApJ, 681, 1341

Whitworth, A. P., Bhattal, A. S., Chapman, S. J., Disney, M. J., \& Turner, J. A. 1994, MNRAS, 268, 291
Wienen, M., Wyrowski, F., Schuller, F., et al. 2012, A\&A, 544, A146

Wilson, T. L., \& Rood, R. 1994, ARA\&A, 32, 191

Wright, E. L., Eisenhardt, P. R. M., Mainzer, A. K., et al. 2010, AJ, 140, 1868

Wu, Y., Liu, T., Meng, F., et al. 2012, ApJ, 756, 76

Yuan, J.-H., Wu, Y., Li, J. Z., \& Liu, H. 2014, ApJ, 797,40

Zavagno, A., Anderson, L. D., Russeil, D., et al. 2010, A\&A, 518, L101

This 2-column preprint was prepared with the AAS $\mathrm{LT}_{\mathrm{E}} \mathrm{X}$ macros v5.2. 